\title{
FILTER GAMES AND PATHOLOGICAL SUBGROUPS OF A COUNTABLE PRODUCT OF LINES
}

\author{
TARAS BANAKH, PETER NICKOLAS ${ }^{\varpi}$ and MANUEL SANCHIS
}

(Received 1 April 2003; revised 6 December 2004)

\author{
Communicated by T. Dooley
}

\begin{abstract}
To each filter $\mathscr{F}$ on $\omega$, a certain linear subalgebra $A(\mathscr{F})$ of $\mathbb{R}^{\omega}$, the countable product of lines, is assigned. This algebra is shown to have many interesting topological properties, depending on the properties of the filter $\mathscr{F}$. For example, if $\mathscr{F}$ is a free ultrafilter, then $A(\mathscr{F})$ is a Baire subalgebra of $\mathbb{R}^{\omega}$ for which the game OF introduced by Tkachenko is undetermined (this resolves a problem of Hernández, Robbie and Tkachenko); and if $\mathscr{F}_{1}$ and $\mathscr{F}_{2}$ are two free filters on $\omega$ that are not near coherent (such filters exist under Martin's Axiom), then $A\left(\mathscr{F}_{1}\right)$ and $A\left(\mathscr{F}_{2}\right)$ are two $o$-bounded and OF-undetermined subalgebras of $\mathbb{R}^{\omega}$ whose product $A\left(\mathscr{F}_{1}\right) \times A\left(\mathscr{F}_{2}\right)$ is OF-determined and not $o$-bounded (this resolves a problem of Tkachenko). It is also shown that the statement that the product of two $o$-bounded subrings of $\mathbb{R}^{\omega}$ is $o$-bounded is equivalent to the set-theoretic principle NCF (Near Coherence of Filters); this suggests that Tkachenko's question on the productivity of the class of $o$-bounded topological groups may be undecidable in ZFC.
\end{abstract}

2000 Mathematics subject classification: primary 03E35, 03E50, 03E60, 22A05, 54A35, 54D80, 54E52, 54G15, 54H11, 54H12, 54H13, $91 \mathrm{~A} 44$.

Keywords and phrases: open-finite game, $o$-bounded group, filter game, near coherence of filters.

\section{Introduction}

In this paper we present a method for constructing examples of topological subgroups, linear sublattices and linear subalgebras of $\mathbb{R}^{\omega}$ which possess various pathological properties. The idea is to assign to a subset $B$ of $\mathbb{R}^{\omega}$ and a filter $\mathscr{F}$ on $\omega$ a special subspace $\circledast(B ; \mathscr{F})$ of $\mathbb{R}^{\omega}$. The algebraic properties of this space $\circledast(B ; \mathscr{F})$ depend on the choice of the set $B$, while the geometric and topological properties of $\circledast(B ; \mathscr{F})$ depend on the choice of the filter $\mathscr{F}$. In particular, if $B$ is the set of all sequences of polynomial growth, then the space $\circledast(B ; \mathscr{F})$, denoted by $A(\mathscr{F})$ in this case, is a linear

(C) 2006 Australian Mathematical Society $1446-7887 / 06 \$ A 2.00+0.00$ 
sublattice and subalgebra of $\mathbb{R}^{\omega}$. If $\mathscr{F}$ is a non-principal ultrafilter, then the algebra $A(\mathscr{F})$ is Baire and OF-undetermined (this example resolves Problem 5 of [11]). If $\mathscr{F}_{1}$ and $\mathscr{F}_{2}$ are two filters on $\omega$ that are not near coherent (such filters exist under Martin's Axiom), then the algebras $A\left(\mathscr{F}_{1}\right)$ and $A\left(\mathscr{F}_{2}\right)$ are $o$-bounded in $\mathbb{R}^{\omega}$, while their product $A\left(\mathscr{F}_{1}\right) \times A\left(\mathscr{F}_{2}\right)$ is not $\varrho$-bounded in $\mathbb{R}^{\omega} \times \mathbb{R}^{\omega}$ and their sum $A\left(\mathscr{F}_{1}\right)+A\left(\mathscr{F}_{2}\right)$ coincides with $\mathbb{R}^{\omega}$ (this answers Problem 3.2 of [22]). On the other hand, the near coherence of all filters on $\omega$ implies that the product $X \times Y$ of any $o$-bounded subrings $X$ and $Y$ of $\mathbb{R}^{\omega}$ is $o$-bounded in $\mathbb{R}^{\omega} \times \mathbb{R}^{\omega}$. This suggests that Problem 3.2 in [22] on the productivity of the class of $o$-bounded topological groups may be equivalent to the principle NCF (Near Coherence of Filters), and hence be independent of ZFC.

To give an idea of our subsequent considerations, we briefly explain the relation of NCF to the productivity problem for the class of $o$-bounded subrings of $\mathbb{R}^{\omega}$ (the definition and basic properties of near coherence will be given later). We shall see in Proposition 2 that for each $o$-bounded subring of $\mathbb{R}^{\omega}$, there is a filter $\mathscr{F}$ with respect to which the subring has a stronger boundedness property that we call $o_{\mathscr{F}}$-boundedness. The latter property, unlike the usual $o$-boundedness, is preserved by products. The classes of $o_{\mathscr{F}}$ - and $o_{\mathscr{F}}$-bounded subsets coincide for near coherent ultrafilters $\mathscr{F}, \mathscr{F}^{\prime}$, and this is the reason why the product of two $o$-bounded subrings of $\mathbb{R}^{\omega}$ is $o$-bounded under NCF.

Now let us recall the definitions of a number of types of boundedness in topological groups. Given a topological group $G$, denote by $\mathscr{N}(e)$ the family of open neighborhoods of the identity $e$ of $G$. A subset $B$ of $G$ is defined to be

- bounded if for any neighborhood $U \in \mathscr{N}(e)$ there is a finite subset $F \subset G$ such that $B \subset F \cdot U$;

- $\sigma$-bounded if $B=\bigcup_{n \in \omega} B_{n}$ is a countable union of bounded subsets $B_{n}$ of $G$;

- $\aleph_{0}$-bounded if for any neighborhood $U \in \mathscr{N}(e)$ there is a subset $F$ of $G$ with $|F| \leq \aleph_{0}$ and $B \subset F \cdot U$;

- o-bounded if for any sequence $\left\{U_{n}\right\}_{n \in \omega} \subset \mathscr{N}(e)$ there is a sequence $\left\{F_{n}\right\}_{n \in \omega}$ of finite subsets of $G$ such that $B \subset \bigcup_{n \in \omega} F_{n} \cdot U_{n}$.

Observe that the condition $B \subset \bigcup_{n \in \omega} F_{n} \cdot U_{n}$ is equivalent to saying that the set $N_{x}=\left\{n \in \omega: x \in F_{n} \cdot U_{n}\right\}$ is non-empty for each $x \in B$. Trying to impose more control on the sets $N_{x}$ for $x \in B$, we arrive at the concept of an $o_{F}$-bounded set. First we introduce some notation. Denote by $\mathscr{P}(\omega)$ (respectively $\left.[\omega]^{\omega}\right)$ the collection of all (infinite) subsets of the set $\omega$ of non-negative integers. Given a family $\mathscr{F} \subset \mathscr{P}(\omega)$ and a function $\varphi: \omega \rightarrow \omega$, let

$$
\varphi[\mathscr{F}]=\{E \subset \omega: \exists F \in \mathscr{F} \text { with } \varphi(F) \subset E\} .
$$

A function $\varphi: \omega \rightarrow \omega$ is called finite-to-one if the pre-image $\varphi^{-1}(n)$ is finite for every $n \in \omega$.

A subset $B$ of a topological group $G$ is called $o_{\mathscr{F}}$-bounded, where $\mathscr{F} \subset \mathscr{P}(\omega)$, if 
for any sequence $\left\{U_{n}\right\}_{n \in \omega} \subset \mathscr{N}(e)$ there is a sequence $\left\{F_{n}\right\}_{n \in \omega}$ of finite subsets of $G$ such that $B \subset \bigcup_{F \in \mathscr{F}} \bigcap_{n \in \varphi(F)} F_{n} \cdot U_{n}$ for some finite-to-one function $\varphi: \omega \rightarrow \omega$. Here we assume conventionally that $\bigcap_{n \in \emptyset} F_{n} \cdot U_{n}=G$, so that every subset of $G$ is $o \mathscr{F}$-bounded if $\emptyset \in \mathscr{F}$.

Observe that a subset $B \subset G$ is $o$-bounded if and only if it is $o_{\mathscr{F}}$-bounded for the collection $\mathscr{F}=\{\{n\}: n \in \omega\}$ of singletons. Note also that any $o_{\mathscr{F}}$-bounded subset $B \subset G$ is $o_{\mathscr{F}}$-bounded for any family $\mathscr{F}^{\prime} \subset \mathscr{P}(\omega)$ and any finite-to-one function $\varphi: \omega \rightarrow \omega$ with $\varphi\left[\mathscr{F}^{\prime}\right] \supset \mathscr{F}$.

It is clear that each $\sigma$-bounded subset $B$ of $G$ is $o$-bounded. In fact, $\sigma$-bounded subsets of $G$ have a stronger property, which is called strict $o$-boundedness in [22] and [10] and II-boundedness in [2]. We define a version of this property parameterised by a collection $\mathscr{F} \subset \mathscr{P}(\omega)$, as follows. Given $\mathscr{F}$, consider the following game $\mathrm{OF}_{\mathscr{F}}$ (abbreviated from Open-Finite) on a subset $B$ of a topological group $G$. Two players, I and II, choose at every step $n \in \omega$ a neighborhood $U_{n} \in \mathscr{N}(e)$ and a finite subset $F_{n} \subset G$, respectively. At the end of the game, II is declared the winner if $B \subset \bigcup_{F \in \mathscr{F}} \bigcap_{n \in \varphi(F)} F_{n} \cdot U_{n}$ for some finite-to-one function $\varphi: \omega \rightarrow \omega$.

A subset $B$ of a topological group $G$ is defined to be

- II $I_{F}$-bounded if the second player has a winning strategy in the game $\mathrm{OF}_{\mathscr{F}}$ on $B$;

- $\mathrm{I}_{\mathscr{F}}$-bounded if the first player has no winning strategy in the game $\mathrm{OF}_{\mathscr{F}}$ on $B$;

- $\mathrm{OF}_{\mathscr{F}}$-determined if one of the players has a winning strategy in the game $\mathrm{OF}_{\mathscr{F}}$ on $B$;

- $\mathrm{OF}_{\mathscr{F}}$-undetermined if $G$ is not $\mathrm{OF}_{\mathscr{F}}$-determined (equivalently, if $G$ is $\mathrm{I}_{\mathscr{F}}$-bounded but not $\mathrm{II}_{\mathscr{F}}$-bounded).

A topological group $G$ is defined to be bounded (respectively $\sigma$-bounded, $\aleph_{0}$-bounded, $\mathrm{II}_{\mathscr{F}}$-bounded, $\mathrm{I}_{\mathscr{F}}$-bounded, of -bounded, $\mathrm{OF}_{\mathscr{F}}$-determined, $\mathrm{OF}_{\mathscr{F}}$-undetermined) if $G$ has the respective property as a subset of $G$. If $\mathscr{F}$ is the collection of all the singletons of $\omega$, then we shall omit the subscript $\mathscr{F}$ and shall speak about the game OF and II-, I-, $o$-bounded, OF-determined and OF-undetermined sets in place of the game $\mathrm{OF}_{\mathscr{F}}$ and $\mathrm{II}_{\mathscr{F}}-, \mathrm{I}_{\mathscr{F}}-, o_{\mathscr{F}}$-bounded, $\mathrm{OF}_{\mathscr{F}}$-determined and $\mathrm{OF}_{\mathscr{F}}$-undetermined sets, respectively. It should be mentioned that in $[1,10,11,22,23]$, II-bounded groups are called 'strictly $o$-bounded', but we prefer the term 'II-bounded', accepted also in [2].

We note that the definitions of all the boundedness conditions above are in fact with respect to the left uniformity of the group $G$. Similar definitions can of course be given with respect to the right uniformity and with respect to the two-sided uniformity. Since however our focus in this paper is almost exclusively on abelian groups, in which these distinctions are irrelevant, we shall work with definitions in the one-sided form given.

Although we have defined the properties of $\mathrm{II}_{\mathscr{F}}-, \mathbf{I}_{\mathscr{F}}$-, and $o_{\mathscr{F}}$-boundedness for arbitrary families $\mathscr{F} \subset \mathscr{P}(\omega)$, they behave especially nicely for the families $\mathscr{F}$ called 
semi-filters.

By a semi-filter, we understand a family $\mathscr{F}$ of infinite subsets of $\omega$ closed under taking supersets and such that $F \backslash K \in \mathscr{F}$ for any $F \in \mathscr{F}$ and any finite subset $K$ of $\omega$. A semi-filter $\mathscr{F}$ is called a filter if $F \cap F^{\prime} \in \mathscr{F}$ for any $F, F^{\prime} \in \mathscr{F}$. It is easy to see that a family of sets is a semi-filter if and only if it is a union of filters. Note that all our filters $\mathscr{F}$ are free in the sense that $\cap \mathscr{F}=\emptyset$. Note also that if $\mathscr{F}$ is a (semi-)filter and $\varphi: \omega \rightarrow \omega$ is a finite-to-one function, then $\varphi[\mathscr{F}]$ is again a (semi-)filter. A filter $\mathscr{F}$ is called an ultrafilter if $\mathscr{F}=\mathscr{F}^{\prime}$ for any filter $\mathscr{F}^{\prime} \supset \mathscr{F}$. Ultrafilters are maximal elements of the naturally ordered set of all filters. This set has a unique minimal element - the Fréchet filter $\mathfrak{F}_{r}$, consisting of all cofinite subsets of $\omega$. The filter $\mathfrak{F}_{r}$ is also the smallest element of the set of all semi-filters, which, unlike the set of all filters, has a unique maximal element - the semi-filter $[\omega]^{\omega}$ consisting of all infinite subsets of $\omega$.

Identifying each subset of $\omega$ with its characteristic function, we identify the powerset $\mathscr{P}(\omega)$ of $\omega$ with the Cantor cube $\{0,1\}^{\omega}$, and thus introduce a metrizable compact topology on $\mathscr{P}(\omega)$. Referring to this topology, we can speak of $\sigma$-compact, meager, analytic or projective subspaces of $\mathscr{P}(\omega)$ or $[\omega]^{\omega}$.

The interplay between the properties of $\mathrm{II}_{\mathscr{F}-}, \mathrm{I}_{\mathscr{F}}-$, and $o_{\mathscr{F}}$-boundedness depends to a large extent on the properties of the family $\mathscr{F}$. We illustrate this thesis by the following diagram, which holds for subsets of $\mathbb{R}^{\omega}$, the countable product of lines (see Theorems 6 and 7). (In fact, this diagram holds more generally for subsets of Lindelöf Cech-complete groups [2].) In the diagram, $\mathscr{F}$ is a filter on $\omega$, while $\mathfrak{F}_{r}$ and $[\omega]^{\omega}$ are the smallest and the largest semi-filters described above, respectively.

$$
\begin{aligned}
& \sigma \text {-bounded } \Leftrightarrow \mathrm{II}_{\mathfrak{F}_{r}} \text {-bounded } \Leftrightarrow \mathrm{I}_{\mathfrak{F}_{r}} \text {-bounded } \Leftrightarrow o_{\mathfrak{F} r} \text {-bounded } \\
& \Downarrow \quad \Downarrow \quad \Downarrow \\
& \mathrm{II}_{\mathscr{F}} \text {-bounded } \Rightarrow \mathrm{I}_{\mathscr{F}} \text {-bounded } \Leftrightarrow o_{\mathscr{F}} \text {-bounded } \\
& \text { 文 } \Downarrow \Downarrow \\
& \mathrm{II}_{[\omega]^{\omega}} \text {-bounded } \Rightarrow \mathrm{I}_{[\omega]^{\omega}} \text {-bounded } \Rightarrow o_{[\omega]^{\omega}} \text {-bounded } \\
& \text { 企 } \Downarrow \\
& \text { II-bounded } \Rightarrow \text { I-bounded } \Rightarrow o \text {-bounded } \Rightarrow \aleph_{0} \text {-bounded }
\end{aligned}
$$

In general, the non-equivalence implications from this diagram cannot be reversed: the countable product of lines $\mathbb{R}^{\omega}$ is $\aleph_{0}$-bounded but not $o$-bounded [10, Example 2.6]; $\mathbb{R}^{\omega}$ contains a dense $G_{\delta}$-subset $\circledast\left([\omega]^{\omega}\right)$ which is $o$-bounded but not I-bounded; this $G_{\delta}$-set provides also an example of an $o_{[\omega]^{\omega}}$-bounded subset which is neither $\mathrm{I}_{[\omega] \omega}$-bounded nor $o_{\mathscr{F}}$-bounded for a filter $\mathscr{F}$ (see Proposition 1); for any nonmeager filter $\mathscr{F}$ the space $\mathbb{R}^{\omega}$ contains a Baire linear subspace $A(\mathscr{F})$ which is $\mathrm{OF}_{\mathscr{F}}$-undetermined, that is, $\mathrm{I}_{\mathscr{F}}$-bounded but not $\mathrm{II}_{\mathscr{F}}$-bounded; this space $A(\mathscr{F})$ is also I-bounded but not II-bounded (see Theorem 8); under the negation of NCF there 
are two linear subspaces $A_{1}, A_{2}$ of $\mathbb{R}^{\omega}$ whose union $A_{1} \cup A_{2}$ is $I_{[\omega]^{\omega}}$-bounded but not $I_{\mathscr{F}}$-bounded for a filter $\mathscr{F}$; these spaces $A_{1}, A_{2}$ are I-bounded in $\mathbb{R}^{\omega}$ but their product $A_{1} \times A_{2}$ is not $o$-bounded in $\mathbb{R}^{\omega} \times \mathbb{R}^{\omega}$ and their sum $A_{1}+A_{2}$ coincides with $\mathbb{R}^{\omega}$ (see Corollary 3 ). Finally, $\mathbb{R}^{\omega}$ contains an I-bounded subspace which is not $I_{[\omega]^{\omega}}$-bounded [2]. Besides these examples (which are all subsets of $\mathbb{R}^{\omega}$ ), there is also an example of a non-metrizable II-bounded group which is not $\sigma$-bounded [10, Example 3.1].

The non-metrizability of the last counterexample is not merely incidental, in view of the following theorem, whose proof can be found in [1, 2] or [20, Section 7].

\section{THEOREM 1. Let $G$ be a metrizable topological group.}

(1) Each II-bounded subset $B$ of $G$ is $\sigma$-bounded.

(2) Each analytic I-bounded subset $B$ of $G$ is $\sigma$-bounded.

(3) If $G$ is an o-bounded SIN-group, then each analytic subset $B$ of $G$ is $\sigma$-bounded.

We recall that a topological group $G$ is called a $S I N$-group if $G$ has a neighborhood base $\mathscr{B}$ at the origin such that $g^{-1} U g=U$ for any $g \in G$ and $U \in \mathscr{B}$.

A topological space $X$ is analytic if it is a metrizable continuous image of a Polish (separable completely metrizable) space. In fact, the class $\Sigma_{1}^{1}$ of analytic spaces is the first member in the hierarchy of projective classes $\Sigma_{n}^{1}$ and $\Pi_{n}^{1}, n \in \mathbb{N}$. These classes are defined by induction. The class $\Pi_{n}^{1}$ consists of all separable metrizable spaces $X$ whose complement $\bar{X} \backslash X$ in some metrizable compactification $\bar{X}$ of $X$ belongs to the class $\Sigma_{n}^{1}$, and the class $\Sigma_{n+1}^{1}$ consists of metrizable continuous images of spaces from the class $\Pi_{n}^{1}$ (see [14, Section 37.A]). Spaces from the class $\bigcup_{n \in \omega} \Sigma_{n}^{1} \cup \Pi_{n}^{1}$ are called projective. It should be mentioned that under the principle of Projective Determinacy [14, Definition 38.15] (which is one of the so-called Strong Set-Theoretic Hypotheses and follows from the existence of a suitable large cardinal [12, page 282], [19]), the analyticity of the subset $B$ in Theorem 1 can be replaced by the projectivity of $B$. All of this shows that examples designed to demonstrate the difference between the boundedness properties we are considering must of necessity have a complex descriptive structure.

A reflection of this is the fact that the first claimed example of a metrizable $o$ bounded non-II-bounded group $H$, presented by Hernández in [10, Example 6.1] (and exploited in [22, page 195], [1, Theorem 4] and [11, Example 2.12]), turned out to be incorrect. (By [1], the group $H$ is analytic, and being non- $\sigma$-bounded, is not $o$-bounded, according to Theorem 1 (3).) The error was noted by the second author; see also [16], [20, page 45]. Valid examples of $o$-bounded non-II-bounded groups have been constructed under certain additional set-theoretic assumptions. In particular, in [11] a (non-metrizable) OF-undetermined group was constructed under the Diamond Principle $\diamond$ (afterwards, a similar example was constructed in ZFC [15] 
or [20, Section 6]). In [1] a Baire OF-undetermined subgroup was constructed in each abelian non-locally compact Polish divisible group under Martin's Axiom. Finally, it was shown in [2] that each abelian non-locally compact Polish group contains an OF-undetermined subgroup (see also [9]). All these examples of OF-undetermined groups were constructed by transfinite induction, and this led naturally to the problem of finding more 'real' and palpable ZFC-examples distinguishing various sorts of boundedness.

In this paper, many such examples are constructed using the filter approach (after writing an initial version of this paper, we learned that a similar filter approach was used also in [16] and [20]). All our examples are subsets of $\mathbb{R}^{\omega}$, the countable product of lines, endowed with the Tychonov product topology. The space $\mathbb{R}^{\omega}$ is a very rich object and carries a wealth of algebraic structures. Besides the linear and group operations, the space $\mathbb{R}^{\omega}$ has the operation of coordinatewise multiplication (that is, $\mathbb{R}^{\omega}$ is a linear topological algebra with unity) as well as the operations of coordinatewise maximum and minimum (that is, $\mathbb{R}^{\omega}$ is a linear topological lattice). We can thus speak of algebraic subobjects of $\mathbb{R}^{\omega}$ of many different types, including subgroups, convex sets, linear subspaces, sublattices, linear subalgebras.

It will be convenient to think of elements of $\mathbb{R}^{\omega}$ as functions from $\omega$ to $\mathbb{R}$. For every $n \in \omega$, consider the seminorm $\|\cdot\|_{n}$ on $\mathbb{R}^{\omega}$ defined by $\|x\|_{n}=\max _{i \leq n}|x(i)|$ for $x \in \mathbb{R}^{\omega}$. Given two functions $f, g \in \mathbb{R}^{\omega}$, we write $f \leq g$ (respectively $f<g$ ) if $f(n) \leq g(n)$ (respectively $f(n)<g(n)$ ) for all $n \in \omega$, and $f \leq^{*} g$ (respectively $f<{ }^{*} g$ ) if there is $m \in \omega$ such that $f(n) \leq g(n)$ (respectively $f(n)<g(n)$ ) for all $n \geq m$.

By the growth of a function $f \in \mathbb{R}^{\omega}$ we understand the function $\uparrow f \in \mathbb{R}^{\omega}$ defined by $\uparrow f(n)=\|f\|_{n}$ for $n \in \omega$. It is clear that $\uparrow f$ is a non-negative non-decreasing function. A subset $A$ of $\mathbb{R}^{\omega}$ is defined to be absolutely symmetric if for any $a \in A$ and $b \in \mathbb{R}^{\omega}$ with $\uparrow b \leq \uparrow a$ we have $b \in A$.

The central objects of our study are the absolutely symmetric subsets of the form

$$
\circledast(B ; \mathscr{F})=\left\{x \in \mathbb{R}^{\omega}: \exists b \in B \exists F \in \mathscr{F} \forall n \in F\|x\|_{n}<\|b\|_{n}\right\},
$$

where $B \subset \mathbb{R}^{\omega}$ and $\mathscr{F}$ is a collection of subsets of $\omega$.

If $B=\{b\}$ for some $b \in \mathbb{R}^{\omega}$, then we write $\circledast(b ; \mathscr{F})$ in place of $\circledast(\{b\} ; \mathscr{F})$. Also, we write $\circledast(\mathscr{F})$ in place of $\circledast($ id; $\mathscr{F})$, where id $\in \mathbb{R}^{\omega}$ is the identity function $i \mapsto i$ for $i \in \omega$. Thus

$$
\circledast(\mathscr{F})=\left\{x \in \mathbb{R}^{\omega}: \exists F \in \mathscr{F} \forall i \in F \quad\|x\|_{i}<i\right\} .
$$

It is clear that if $B \subset B^{\prime}$ and $\mathscr{F} \subset \mathscr{F}^{\prime}$, then $\circledast(B ; \mathscr{F}) \subset \circledast\left(B^{\prime} ; \mathscr{F}^{\prime}\right)$. If the collection $\mathscr{F}$ consists of infinite subsets of $\omega$, then the set $\circledast(B ; \mathscr{F})$ can be equivalently defined as

$$
\circledast(B ; \mathscr{F})=\left\{x \in \mathbb{R}^{\omega}: \exists b \in B \exists F \in \mathscr{F} \text { with }|x|<b_{F}\right\},
$$


where $b_{F} \in \mathbb{R}^{\omega}$ is the non-decreasing step-function assigning to each $i \in \omega$ the number $b_{F}(i)=\min \left\{\|b\|_{j}: j \in F \cap[i, \infty)\right\}=\|b\|_{\min (F \cap[i, \infty))}$.

For families $\mathscr{F}_{1}, \ldots, \mathscr{F}_{n} \subset \mathscr{P}(\omega)$ and a function $b \in \mathbb{R}^{\omega}$, define by induction the sets

$$
\circledast\left(b ; \mathscr{F}_{1}, \ldots, \mathscr{F}_{i+1}\right)=\circledast\left(\circledast\left(b ; \mathscr{F}_{1}, \ldots, \mathscr{F}_{i}\right) ; \mathscr{F}_{i+1}\right)
$$

for $i<n$. We shall write $\circledast\left(\mathscr{F}_{1}, \ldots, \mathscr{F}_{n}\right)$ in place of $\circledast\left(\right.$ id; $\left.\mathscr{F}_{1}, \ldots, \mathscr{F}_{n}\right)$. It is easy to see that $\circledast(b ; \mathscr{F}, \ldots, \mathscr{F})=\circledast(b ; \mathscr{F})$ whenever the family $\mathscr{F}$ is a filter.

The sets of the form $\circledast(b ; \mathscr{F})$ play a fundamental role in the class of $o_{\mathscr{F}}$-bounded subsets of $\mathbb{R}^{\omega}$.

THEOREM 2. (1) A subset $B \subset \mathbb{R}^{\omega}$ is $o_{\mathscr{F}}$-bounded, where $\mathscr{F}$ is a semi-filter on $\omega$, if and only if $B \subset \circledast(b ; \varphi[\mathscr{F}])$ for some increasing function $b: \omega \rightarrow \omega$ and some finite-to-one map $\varphi: \omega \rightarrow \omega$.

(2) A subset $B \subset \mathbb{R}^{\omega}$ is o-bounded if and only if $B$ is $o_{[\omega]^{\omega}}$-bounded if and only if $B \subset \circledast\left(b ;[\omega]^{\omega}\right)$ for some $b \in \mathbb{R}^{\omega}$.

Our interest in subsets of the form $\circledast(B ; \mathscr{F})$ can be explained by the following theorem, which describes some algebraic and geometric properties of such sets, and follows easily from the corresponding definitions.

THEOREM 3. Let $B \subset \mathbb{R}^{\omega}$ and let $\mathscr{F}$ be a filter on $\omega$.

(1) If $\sup _{x \in B, n \in \omega}\|x\|_{n}=\infty$, then $\circledast(B ; \mathscr{F})$ is a dense subset of $\mathbb{R}^{\omega}$.

(2) If for all $x, y \in B$ and $t \in[0,1]$ there exists $z \in B$ with $t \uparrow x+(1-t) \uparrow y \leq^{*} \uparrow z$, then $\circledast(B ; \mathscr{F})$ is a convex subset of $\mathbb{R}^{\omega}$.

(3) If for all $x, y \in B$ there exists $z \in B$ with $\max \{\uparrow x, \uparrow y\} \leq^{*} \uparrow z$, then $\circledast(B ; \mathscr{F})$ is a sublattice of $\mathbb{R}^{\omega}$.

(4) If for all $x, y \in B$ there exists $z \in B$ with $\uparrow x+\uparrow y \leq^{*} \uparrow z$, then $\circledast(B$; $\mathscr{F})$ is a linear sublattice of $\mathbb{R}^{\omega}$.

(5) If for all $x, y \in B$ there exists $z \in B$ with $\uparrow(x \cdot y)+\uparrow x+\uparrow y \leq{ }^{*} \uparrow z$, then $\circledast(B ; \mathscr{F})$ is a linear subalgebra of $\mathbb{R}^{\omega}$.

Next, we investigate the dependence of the topological and boundedness properties of the set $\circledast(B ; \mathscr{F})$ on the properties of the set $B$ and the semi-filter $\mathscr{F}$.

THEOREM 4. Let $B \subset \mathbb{R}^{\omega}$ and let $\mathscr{F}$ be a semi-filter on $\omega$.

(1) If the set $B$ is $\sigma$-bounded in $\mathbb{R}^{\omega}$ (and $\mathscr{F}$ is a filter), then the set $\circledast(B ; \mathscr{F})$ is $o_{\mathscr{F}}$-bounded (and $I_{\mathscr{F}}$-bounded) in $\mathbb{R}^{\omega}$.

(2) If $\circledast(B ; \mathscr{F}) \neq \mathbb{R}^{\omega}$, then $B$ is o-bounded in $\mathbb{R}^{\omega}$.

(3) If the space $B$ is analytic and $\mathscr{F}$ is a non-meager filter, then $\circledast(B ; \mathscr{F})$ is $o_{\mathscr{F}}$-bounded if and only if $\circledast(B ; \mathscr{F}) \neq \mathbb{R}^{\omega}$ if and only if $B$ is $\sigma$-bounded. 
(4) The set $\circledast(B ; \mathscr{F})$ is $\sigma$-bounded in $\mathbb{R}^{\omega}$ if and only if either $\sup _{n \in \omega}\|b\|_{n}<\infty$ for each $b \in B$ or $B$ is $\sigma$-bounded and $\mathscr{F}$ is meager in $[\omega]^{\omega}$.

Next, we study the topological and descriptive structure of the sets $\circledast(B ; \mathscr{F})$. We recall that a topological space $X$ is Baire if the intersection of any countable family of open dense subsets of $X$ is dense in $X$; we say that $X$ is hereditarily Baire if each closed subspace of $X$ is Baire. It is known that each Polish space is hereditarily Baire and that a coanalytic space (that is, a space of the projective class $\Pi_{1}^{1}$ ) is hereditarily Baire if and only if it is Polish (see [14, Corollary 21.21]).

THEOREM 5. Let $B \subset \mathbb{R}^{\omega}$ and let $\mathscr{F}$ be a semi-filter on $\omega$.

(1) The set $\circledast(B ; \mathscr{F})$ is a continuous image of $B \times \mathscr{F} \times(-1,1)^{\omega}$.

(2) If $B, \mathscr{F} \in \Sigma_{n}^{1}$ for some $n \in \mathbb{N}$, then $\circledast(B ; \mathscr{F}) \in \Sigma_{n}^{1}$.

(3) If $\mathscr{F}$ is a non-meager filter on $\omega$, then the space $\circledast(\mathscr{F})$ is Baire; moreover, the closure $\bar{A}$ of any subset $A \subset \circledast\left(\mathfrak{F}_{r}\right)$ in $\circledast(\mathscr{F})$ is a Baire space.

(4) If $\mathscr{F}$ is a non-meager $P$-filter, then the space $\circledast(\mathscr{F})$ is hereditarily Baire.

(5) The space $\circledast(B ; \mathscr{F})$ is not hereditarily Baire if $B$ contains a function sequence $\left(b_{n}\right)_{n \in \omega}$ with $\uparrow b_{n}<^{*} \uparrow b_{n+1}$ for every $n \in \omega$ and such that for every $b \in B$ there is $n \in \omega$ such that $\uparrow b \leq^{*} \uparrow b_{n}$.

It is well-known that each ultrafilter on $\omega$ is non-meager (see [14, Exercise 8.50] or [26]). Moreover, there are models of ZFC containing non-meager filters of projective class $\Sigma_{3}^{1}$ (see [3] or [13]). Repeating the argument of Talagrand [21] (see also [24, page 32]), we can prove that a semi-filter $\mathscr{F}$ on $\omega$ is meager if and only if $\mathscr{F}$ lies in a $\sigma$-compact subset of $[\omega]^{\omega}$ if and only if there is an increasing number sequence $\left(m_{k}\right)_{k \in \omega} \in \omega^{\omega}$ such that each $F \in \mathscr{F}$ meets all but finitely many intervals $\left[m_{k}, m_{k+1}\right)$. A filter $\mathscr{F}$ is called a $P$-filter if for any countable subcollection $\mathscr{F}^{\prime} \subset \mathscr{F}$ there is $F \in \mathscr{F}$ such that the complement $F \backslash F^{\prime}$ is finite for any $F^{\prime} \in \mathscr{F}^{\prime}$. Ultrafilters that are $P$-filters are called $P$-points. It is well known that $P$-points exist under Martin's Axiom, while there exist models of ZFC without $P$-points [26]. Let us note that the Fréchet filter $\mathfrak{F}_{r}$ of all cofinite subsets of $\omega$ is a meager $P$-filter. The problem of the existence of a non-meager $P$-filter seems to still be open (see [4, page 230]).

Theorems 2 and 4 will allow us to prove the following two important results describing the interplay between various boundedness properties.

THEOREM 6. For a subset $B \subset \mathbb{R}^{\omega}$, the following conditions are equivalent:

(1) B is $\sigma$-bounded;

(2) B is II-bounded;

(3) $B$ is $I_{\mathscr{F}}$-bounded for some semi-filter $\mathscr{F}$;

(4) $B$ is $I_{\mathscr{F}}$-bounded for any semi-filter $\mathscr{F}$;

(5) $B$ is $o_{\mathscr{F}}$-bounded for some meager semi-filter $\mathscr{F}$. 
THEOREM 7. (1) If $\mathscr{F}$ is a filter, then each of-bounded subset of $\mathbb{R}^{\omega}$ is $I_{\mathscr{F}}$ bounded.

(2) For any filters $\mathscr{F}_{1}, \ldots, \mathscr{F}_{n}$ and any function $b \in \mathbb{R}^{\omega}$, the union $\circledast\left(b ; \mathscr{F}_{1}\right) \cup \cdots \cup$ $\circledast\left(b ; \mathscr{F}_{n}\right)$ is $I_{[\omega]^{\omega}}$-bounded in $\mathbb{R}^{\omega}$.

Now we apply Theorems 4 and 5 to sets of the form $\circledast(\mathscr{F})$.

COROLlaRY 1. Let $\mathscr{F}$ be a semi-filter on $\omega$.

(1) $\circledast(\mathscr{F})$ is an absolutely symmetric dense of -bounded subset of $\mathbb{R}^{\omega}$.

(2) If $\mathscr{F}$ is a filter, then $\circledast(\mathscr{F})$ is a $I_{\mathscr{F}}$-bounded convex sublattice of $\mathbb{R}^{\omega}$.

(3) $\circledast(\mathscr{F})$ is $\sigma$-bounded in $\mathbb{R}^{\omega}$ if and only if the semi-filter $\mathscr{F}$ is meager.

(4) If $\mathscr{F}$ is a non-meager filter on $\omega$, then the set $\circledast(\mathscr{F})$ is $O F_{\mathscr{F}}$-undetermined and Baire.

(5) If $\mathscr{F}$ is a non-meager P-filter, then the space $\circledast(\mathscr{F})$ is hereditarily Baire.

In the case when $\mathscr{F}=[\omega]^{\omega}$, the set $\circledast(\mathscr{F})$ gives us an interesting example of an o-bounded subset.

PROPOSITION 1. The set $\circledast\left([\omega]^{\omega}\right)$ has the following properties.

(1) $\circledast\left([\omega]^{\omega}\right)$ is a dense absolutely symmetric $G_{\delta}$-subset of $\mathbb{R}^{\omega}$.

(2) $\circledast\left([\omega]^{\omega}\right)$ is o-bounded and $o_{[\omega]}$-bounded.

(3) $\circledast\left([\omega]^{\omega}\right)$ is not I-bounded.

(4) For any filter $\mathscr{F}$, the set $\circledast\left([\omega]^{\omega}\right)$ is not $o_{\mathscr{F}}$-bounded.

(5) The product $B \times \circledast\left([\omega]^{\omega}\right)$ with a subset $B \subset \mathbb{R}^{\omega}$ is o-bounded in $\mathbb{R}^{\omega} \times \mathbb{R}^{\omega}$ if and only if $B$ is $\sigma$-bounded in $\mathbb{R}^{\omega}$.

As observed earlier, we have $\circledast(\circledast(\mathscr{F}) ; \mathscr{F})=\circledast(\mathscr{F}, \mathscr{F})=\circledast(\mathscr{F})$ for any filter $\mathscr{F}$. If $\mathscr{F}$ is a non-meager filter, then by Corollary 1 (4) the set $B=\circledast(\mathscr{F})$ is $\mathrm{OF}_{\mathscr{F}}$ undetermined, that is, $\mathrm{I}_{\mathscr{F}}$-bounded but not $\mathrm{I}_{\mathscr{F}}$-bounded. In particular, $B$ is not $\sigma$-bounded, while $\circledast(B ; \mathscr{F})=\circledast(\mathscr{F})=B$ is $\mathrm{I}_{\mathscr{F}}$-bounded. This shows that the analyticity of the set $B$ in Theorem 4 (3) is essential.

Now we apply Theorems $3-5$ to subsets of the form $A(\mathscr{F})=\circledast\left(\left\{\mathrm{id}^{n}\right\}_{n \in \omega} ; \mathscr{F}\right)$, where $\operatorname{id}^{n}$ is the map $i \mapsto i^{n}$ for $i \in \omega$. Note that when $\mathscr{F}=\mathfrak{F}_{r}$, the set $A(\mathscr{F})$ coincides with the set of all functions of polynomial growth. In the particular case of the sets $A(\mathscr{F})$, Theorems 3-5 imply the following.

THEOREM 8. Let $F$ be a filter on $\omega$.

(1) $A(\mathscr{F})$ is a dense absolutely symmetric linear sublattice and subalgebra of $\mathbb{R}^{\omega}$.

(2) $A(\mathscr{F})$ is a $I_{\mathscr{F}}$-bounded subset of $\mathbb{R}^{\omega}$.

(3) If $\mathscr{F}$ is meager, then $A(\mathscr{F})$ is a $\sigma$-bounded subset of $\mathbb{R}^{\omega}$.

(4) If $\mathscr{F}$ is non-meager, then $A(\mathscr{F})$ is a Baire OF $\mathscr{F}$-undetermined subset of $\mathbb{R}^{\omega}$. 
(5) If $\mathscr{F}$ is a non-meager $P$-filter, then $A(\mathscr{F})$ contains the absolutely symmetric dense convex hereditarily Baire sublattice $\circledast(\mathscr{F})$.

(6) The space $A(\mathscr{F})$ is not hereditarily Baire.

(7) The space $A(\mathscr{F})$ is a continuous image of $\mathscr{F} \times \mathbb{N} \times(-1,1)^{\omega}$.

(8) If $\mathscr{F} \in \Sigma_{n}^{1}$ for some $n \in \mathbb{N}$, then $A(\mathscr{F}) \in \Sigma_{n}^{1}$.

Since each ultrafilter is non-meager [26] and there are models of ZFC containing non-meager filters of projective class $\Sigma_{3}^{1}$ (see [3] or [13]), Theorems 8 implies the following corollary, resolving Problem 5 of [11].

COROLLARY 2. The countable product $\mathbb{R}^{\omega}$ contains a Baire OF-undetermined linear subalgebra. It is consistent to assume that this subalgebra belongs to the projective class $\Sigma_{3}^{1}$.

It is interesting to remark that under the principle of Projective Determinacy, each projective subset of a Polish group is OF-determined (this follows [1, Proposition 4] and [14, Exercise 38.18]). Thus the existence of a projective OF-undetermined subset of $\mathbb{R}^{\omega}$ is undecidable in ZFC.

Next, we consider Problem 3.2 of [22], concerning products of (strictly) $o$-bounded groups. Answering a part of this problem, it was shown in [2] that the product $G \times H$ of two II-bounded topological groups is II-bounded. Moreover, the product $G \times H$ of a II-bounded group $G$ and an $o$-bounded group $H$ is $o$-bounded. For products of Ior $o$-bounded groups the situation is different. Assuming the existence of two filters that are not near coherent, we shall construct two I-bounded subalgebras of $\mathbb{R}^{\omega}$ whose product is not $o$-bounded in $\mathbb{R}^{\omega} \times \mathbb{R}^{\omega}$ and whose sum in $\mathbb{R}^{\omega}$ coincides with $\mathbb{R}^{\omega}$.

Families $\mathscr{F}_{1}, \ldots, \mathscr{F}_{n} \subset \mathscr{P}(\omega)$ are called near coherent if there is an increasing number sequence $\left(m_{k}\right)_{k \in \omega} \in \omega^{\omega}$ such that for any elements $F_{1} \in \mathscr{F}_{1}, \ldots, F_{n} \in \mathscr{F}_{n}$ there is $k \in \omega$ such that $\left[m_{k-1}, m_{k+1}\right) \cap F_{i} \neq \emptyset$ for all $i \leq n$. Near coherence of filters was introduced and studied in detail by Blass in $[5,6]$. The statement that any two filters on $\omega$ are near coherent is known in set theory as NCF; NCF is false under Martin's Axiom [5], but there are models of ZFC in which NCF is true [7, 8]. Note that the Talagrand characterization [21] of meagerness quoted earlier implies that meager (semi-)filters $\mathscr{F}_{1}, \ldots, \mathscr{F}_{n}$ are near coherent.

THEOREM 9. For (semi-)filters $\mathscr{F}_{1}, \ldots, \mathscr{F}_{n}$, the following conditions are equivalent.

(1) The (semi-)filters $\mathscr{F}_{1}, \ldots, \mathscr{F}_{n}$ are near coherent.

(2) For any function $b \in \mathbb{R}^{\omega}$ the product $\circledast\left(b ; \mathscr{F}_{1}\right) \times \cdots \times \circledast\left(b ; \mathscr{F}_{n}\right)$ is $o_{\mathscr{F}}$-bounded in $\left(\mathbb{R}^{\omega}\right)^{n}$ for some (semi-) filter $\mathscr{F}$.

(3) The product $A\left(\mathscr{F}_{1}\right) \times \cdots \times A\left(\mathscr{F}_{n}\right)$ is o-bounded in $\left(\mathbb{R}^{\omega}\right)^{n}$.

(4) The product $\circledast\left(\mathscr{F}_{1}\right) \times \cdots \times \circledast\left(\mathscr{F}_{n}\right)$ is o-bounded in $\left(\mathbb{R}^{\omega}\right)^{n}$. 
(5) The sum $\circledast\left(\mathscr{F}_{1}\right)+\cdots+\circledast\left(\mathscr{F}_{n}\right)$ is o-bounded in $\mathbb{R}^{\omega}$.

(6) $\circledast\left(\mathscr{F}_{1}\right)+\cdots+\circledast\left(\mathscr{F}_{n}\right) \neq \mathbb{R}^{\omega}$.

Moreover, if $\mathscr{F}_{1}, \ldots, \mathscr{F}_{n}$ are filters, then the conditions (1)-(6) are equivalent to the following.

(7) There is a permutation $\sigma$ of $\{1, \ldots, n\}$ such that for any function $b \in \mathbb{R}^{\omega}$ the set $\circledast\left(b ; \mathscr{F}_{\sigma(1)}, \ldots, \mathscr{F}_{\sigma(n)}\right)$ is $o_{\mathscr{F}}$-bounded for some filter $\mathscr{F}$.

(8) There is a permutation $\sigma$ of $\{1, \ldots, n\}$ such that $\circledast\left(\mathscr{F}_{\sigma(1)}, \ldots, \mathscr{F}_{\sigma(n)}\right) \neq \mathbb{R}^{\omega}$.

(9) The union $\circledast\left(\mathscr{F}_{1}\right) \cup \cdots \cup \circledast\left(\mathscr{F}_{n}\right)$ is $o_{\mathscr{F}}$-bounded for some filter $\mathscr{F}$.

Theorems 9 and 7 imply the following.

COROLLARY 3. Under the negation of NCF, there are two I-bounded subalgebras $A_{1}, A_{2}$ of $\mathbb{R}^{\omega}$ such that

(1) the product $A_{1} \times A_{2}$ is not o-bounded in $\mathbb{R}^{\omega} \times \mathbb{R}^{\omega}$;

(2) the sum $A_{1}+A_{2}$ coincides with $\mathbb{R}^{\omega}$;

(3) the union $A_{1} \cup A_{2}$ is $I_{[\omega]^{\omega}}$-bounded in $\mathbb{R}^{\omega}$;

(4) the union $A_{1} \cup A_{2}$ fails to be $o_{F}$-bounded for all filters $\mathscr{F}$.

After writing this paper, we learned that a result similar to Corollary 3 had been obtained in [16] and [20, Section 5]. Specifically, under $\mathrm{CH}$ two $o$-bounded linear subspaces $L_{1}, L_{2} \subset \mathbb{R}^{\omega}$ were constructed whose sum $L_{1}+L_{2}$ equals $\mathbb{R}^{\omega}$. In fact, the spaces $L_{1}, L_{2}$ have the Menger property, which is stronger than $o$-boundedness. Another result of this sort can be also found in [25].

It turns out that additional set-theoretic assumptions of some kind, such as those in [16] and Corollary 3, are essential: under NCF the product of two $o$-bounded subrings of $\mathbb{R}^{\omega}$ is $o$-bounded. Indeed, this result is true for what we call mixable subsets of $\mathbb{R}^{\omega}$.

We shall say that a subset $X$ of $\mathbb{R}^{\omega}$ is mixable if there exists a non-decreasing function $f:[0, \infty) \rightarrow[0, \infty)$ such that for any $x, y \in X$ there is $z \in X$ such that $f \circ \uparrow z \geq^{*} \max \{\uparrow x, \uparrow y\}$. Many examples of mixable subsets of $\mathbb{R}^{\omega}$ are supplied by subsets of $\mathbb{R}^{\omega}$ closed with respect to certain algebraic operations. For example, any subring of $\mathbb{R}^{\omega}$ is mixable, because $\sqrt{x^{2}+y^{2}} \geq \max \{|x|,|y|\}$ for any $x, y \in \mathbb{R}^{\omega}$. An additive subgroup $G$ of $\mathbb{R}^{\omega}$ is mixable if it is $|\cdot|$-closed, that is, if $|x| \in G$ for each $x \in G$. In its turn, since $|x|=\max \{x,-x\}$, each sublattice $L$ of $\mathbb{R}^{\omega}$ is $|\cdot|$-closed provided it is centrally symmetric in the sense that $-x \in L$ for each $x \in L$. We do not know however if every additive subgroup of $\mathbb{R}^{\omega}$ is mixable. Mixable $o$-bounded subsets of $\mathbb{R}^{\omega}$ have the following remarkable property.

PROPOSITION 2. A mixable subset $X \subset \mathbb{R}^{\omega}$ is o-bounded in $\mathbb{R}^{\omega}$ if and only if it is $o_{\mathscr{F}}$-bounded in $\mathbb{R}^{\omega}$ for some filter $\mathscr{F}$ on $\omega$. 
This proposition will help us to characterize NCF in terms of preservation of $o$ boundedness by products. It is interesting to compare our characterization (resulting from Theorems 2, 7,9) with the characterizations of NCF presented in [6].

THEOREM 10. The following conditions are equivalent.

(1) NCF is true (that is, any two filters on $\omega$ are near coherent).

(2) The product $X \times Y$ of o-bounded mixable subsets $X, Y \subset \mathbb{R}^{\omega}$ is $I_{\mathscr{F}}$-bounded in $\mathbb{R}^{\omega} \times \mathbb{R}^{\omega}$ for some filter $\mathscr{F}$.

(3) The product $X \times Y$ of o-bounded centrally symmetric sublattices $X, Y \subset \mathbb{R}^{\omega}$ is $I_{\mathscr{F}}$-bounded in $\mathbb{R}^{\omega} \times \mathbb{R}^{\omega}$ for some filter $\mathscr{F}$.

(4) The product $X \times Y$ ofo-bounded $|\cdot|$-closed sublattices $X, Y \subset \mathbb{R}^{\omega}$ is $I_{\mathscr{F}}$-bounded in $\mathbb{R}^{\omega} \times \mathbb{R}^{\omega}$ for some filter $\mathscr{F}$.

(5) The product $X \times Y$ of o-bounded $|\cdot|$-closed additive subgroups $X, Y \subset \mathbb{R}^{\omega}$ is $I_{\mathscr{F}}$-bounded in $\mathbb{R}^{\omega} \times \mathbb{R}^{\omega}$ for some filter $\mathscr{F}$.

(6) The product $X \times Y$ of o-bounded subrings $X, Y \subset \mathbb{R}^{\omega}$ is $I_{\mathscr{F}}$-bounded in $\mathbb{R}^{\omega} \times \mathbb{R}^{\omega}$ for some filter $\mathscr{F}$.

(7) The product $X \times Y$ of o-bounded linear subalgebras $X, Y \subset \mathbb{R}^{\omega}$ is $I_{\mathcal{F}}$-bounded in $\mathbb{R}^{\omega} \times \mathbb{R}^{\omega}$ for some filter $\mathscr{F}$.

(8) For any filters $\mathscr{F}_{1}, \mathscr{F}_{2}$ on $\omega$, the product $A\left(\mathscr{F}_{1}\right) \times A\left(\mathscr{F}_{2}\right)$ of the I-bounded subalgebras $A\left(\mathscr{F}_{1}\right), A\left(\mathscr{F}_{2}\right)$ of $\mathbb{R}^{\omega}$ is o-bounded in $\mathbb{R}^{\omega} \times \mathbb{R}^{\omega}$.

(9) For any filters $\mathscr{F}_{1}, \mathscr{F}_{2}$ on $\omega$, the sum $A\left(\mathscr{F}_{1}\right)+A\left(\mathscr{F}_{2}\right) \neq \mathbb{B}^{\omega}$.

Finally, let us ask a question suggested by the above results. We have explored subrings of $\mathbb{R}^{\omega}$, but we know nothing about subrings of the countable product $\mathbb{C}^{\omega}$ of the complex plane $\mathbb{C}$.

QUESTION 1. Is every o-bounded subgroup (subring) of $\mathbb{C}^{\omega}$ mixable? l-bounded? $o_{\mathscr{F}}$-bounded for some filter $\mathscr{F}$ ?

\section{Filter games and properties of the sets $\circledast(B ; \mathscr{F})$}

Now let us pass to proofs of our results. We start from the proofs of certain statements concerning the interplay between the game $\mathrm{OF}_{\mathscr{F}}$ on subsets of $\mathbb{R}^{\omega}$ and the filter games considered in [17] and [18].

First we make precise the notions of a game and of a strategy in a game. From the most general point of view, the games we consider in this paper can be described as follows. Suppose that we are supplied with sets $X, Y$ and a subset $\Phi$ of the product $X^{\omega} \times Y^{\omega}$ ( $\Phi$ can be thought of as some property of pairs of sequences $\left.\left(\left(x_{n}\right),\left(y_{n}\right)\right) \in X^{\omega} \times Y^{\omega}\right)$. Two players, I and II, choose at every step $n \in \omega$ a point $x_{n}$ of the set $X$ and a point $y_{n}$ of the set $Y$, respectively. At the end of the 
game, player II is declared the winner if the constructed sequences $\left(x_{n}\right)_{n \in \omega}$ and $\left(y_{n}\right)_{n \in \omega}$ have the property $\Phi$ (equivalently, the pair $\left(\left(x_{n}\right)_{n \in \omega},\left(y_{n}\right)_{n \in \omega}\right)$ belongs to the set $\Phi$ ); otherwise, player I is declared the winner. The sets $X$ and $Y$ are called the sets of admissible moves of I and II, respectively. Thus the game can be identified with the subset $\Phi \subset X^{\omega} \times Y^{\omega}$.

A strategy of II in the game $\Phi \subset X^{\omega} \times Y^{\omega}$ can be thought as a function $\$_{\text {II }}: X^{<\omega} \rightarrow Y$, where $X^{<\omega}=\bigcup_{n \in \omega} X^{n}$ stands for the family of all finite sequences of elements of $X$ (including the empty sequence). Player II plays according to a strategy $\$_{\text {II }}: X^{<\omega} \rightarrow Y$ if $y_{k}=\$_{\text {II }}\left(x_{0}, \ldots, x_{k}\right)$ for each $k \in \omega$ and each $\left(x_{0}, \ldots x_{k}\right) \in X^{k+1}$. A strategy $\$_{\text {II }}: X^{<\omega} \rightarrow Y$ of II is winning in a game $\Phi \subset X^{\omega} \times Y^{\omega}$ if for any infinite sequence $\left(x_{n}\right)_{n \in \omega} \in X^{\omega}$ the pair $\left(\left(x_{n}\right)_{n \in \omega},\left(y_{n}\right)_{n \in \omega}\right)$ belongs to $\Phi$ whenever II plays according to the strategy $\$_{\mathrm{II}}$.

Dually, a strategy of $I$ in a game $\Phi \subset X^{\omega} \times Y^{\omega}$ is a function $\$_{1}: Y^{<\omega} \rightarrow X$, and player I plays according to a strategy $\$_{\mathrm{I}}$ if $x_{k}=\$_{\mathrm{I}}\left(y_{0}, \ldots, y_{k-1}\right)$ for each $k \in \omega$ and $\left(y_{0}, \ldots, y_{k-1}\right) \in Y^{k}$. A strategy $\$_{\mathrm{I}}: Y^{<\omega} \rightarrow X$ of $\mathrm{I}$ is winning if for any infinite sequence $\left(y_{k}\right)_{k \in \omega} \in Y^{\omega}$ the pair $\left(\left(x_{n}\right)_{n \in \omega},\left(y_{n}\right)_{n \in \omega}\right)$ fails to belong to $\Phi$ whenever I plays according to the strategy $\$_{\mathrm{I}}$.

Let $X$ be a set. For a finite sequence $\sigma=\left(x_{0}, \ldots, x_{n}\right) \in X^{<\omega}$ and a point $x \in X$, let $|\sigma|$ denote the length $n+1$ of $\sigma$ and write $\sigma^{\wedge} x=\left(x_{0}, \ldots, x_{n}, x\right)$. By $[X]^{\omega}$ we denote the family of all infinite subsets of $X$ and by $[X]^{<\omega}=\mathscr{P}(X) \backslash[X]^{\omega}$ the family of all finite subsets of $X$.

We shall reduce our game $\mathrm{OF}_{\mathscr{F}}$ to the game $\mathfrak{G}\left(\mathscr{X},[\omega]^{<\omega}, \mathscr{Z}\right)$ considered in [17] and [18]. Given subsets $\mathscr{X}, \mathscr{Z} \subset \mathscr{P}(\omega)$, the game $\mathfrak{G}\left(\mathscr{X},[\omega]^{<\omega}, \mathscr{Z}\right)$ is defined as follows. At every step $k \in \omega$, two players, I and II, choose an element $X_{k} \in \mathscr{X}$ and a finite subset $s_{k} \subset X_{k}$, respectively. At the end of the game, II is declared the winner if $\bigcup_{k \in \omega} s_{k} \in \mathscr{Z}$.

We shall exploit the following two results proven in [17, Theorems 2.11 and 2.15]. (We recall that $\mathfrak{F}_{r}$ stands for the Fréchet filter of all cofinite subsets of $\omega$.)

LEMMA 1 (Laflamme [17]). Let $\mathscr{F}$ be a filter on $\omega$.

(1) I has a winning strategy in the game $\mathfrak{G}\left(\mathfrak{F}_{r},[\omega]^{<\omega}, \mathscr{F}\right)$ if and only if the filter $\mathscr{F}$ is meager.

(2) I has no winning strategy in the game $\mathfrak{G}\left(\mathscr{F},[\omega]^{<\omega}, \mathscr{F}\right)$ if and only if $\mathscr{F}$ is a non-meager $P$-filter.

To reduce the game $\mathrm{OF}_{\mathscr{F}}$ on $\circledast(b ; \mathscr{F})$ to the game $\mathfrak{G}\left(\mathfrak{F}_{r},[\omega]^{<\omega}, \mathscr{F}\right)$, we shall consider two intermediate games: $\mathrm{OC}_{\mathscr{F}}$ (abbreviated from Open-Compact) and $\mathrm{LH}_{\mathscr{F}}$ (abbreviated from Length-Height) on subsets of $\mathbb{R}^{\omega}$.

For a family $\mathscr{F} \subset \mathscr{P}(\omega)$, the game $\mathrm{OC}_{\mathscr{F}}$ on a subset $B$ of a topological group $G$ is defined as follows. Two players, I and II, at every step $n \in \omega$ choose a neighborhood 
$U_{n} \in \mathscr{N}(e)$ and a compact subset $K_{n}$ of $G$, respectively. At the end of the game, II is declared the winner if $B \subset \bigcup_{F \in \mathscr{F}} \bigcap_{n \in \varphi(F)} K_{n} \cdot U_{n}$ for some finite-to-one function $\varphi: \omega \rightarrow \omega$ (recall that $\mathscr{N}(e)$ is the family of all neighborhoods of the identity $e$ of the group $G)$. We denote by $\mathscr{K}(G)$ the collection of all compact subsets of $G$.

LEMMA 2. For a family $\mathscr{F} \subset \mathscr{P}(\omega)$, the player I has a winning strategy in the game $O F_{\mathscr{F}}$ on a subset $B$ of a topological group $G$ if and only if $I$ has a winning strategy in the game $O C_{\mathscr{F}}$ on $B$.

PROOF. The 'if' part is trivial and follows from the compactness of finite subsets of $G$. To prove the 'only if' part, assume that $\$:\left([G]^{<\omega}\right)^{<\omega} \rightarrow \mathscr{N}(e)$ is a winning strategy of $\mathrm{I}$ in the game $\mathrm{OF}_{\mathscr{F}}$ on $B$. Fix a function $g: \mathscr{N}(e) \rightarrow \mathscr{N}(e)$ such that $g(U) \cdot g(U) \subset U$ for any $U \in \mathscr{N}(e)$ and, using compactness, fix a function $f: \mathscr{K}(G) \times \mathscr{N}(e) \rightarrow[G]^{<\omega}$ such that $K \subset f(K, U) U$ for any $(K, U) \in \mathscr{K}(G) \times$ $\mathscr{N}(e)$.

We define a strategy $\overline{\$}: \mathscr{K}(G)^{<\omega} \rightarrow \mathscr{N}(e)$ of I in the game $\mathrm{OC}_{\mathscr{F}}$ by induction, by setting $\overline{\$}(\emptyset)=g(\$(\emptyset))$ and

$$
\overline{\$}\left(K_{0}, \ldots, K_{n}\right)=g \circ \$\left(f\left(K_{0}, \overline{\$}(\emptyset)\right), f\left(K_{1}, \overline{\$}\left(K_{0}\right)\right), \ldots, f\left(K_{n}, \overline{\$}\left(K_{0}, \ldots, K_{n-1}\right)\right)\right)
$$

for $\left(K_{0}, \ldots, K_{n}\right) \in \mathscr{K}(G)^{<\omega}$. Let us show that $\overrightarrow{\$}$ is a winning strategy. Fix any infinite sequence $\left(K_{n}\right)_{n \in \omega} \in \mathscr{K}(G)^{\omega}$ of compact subsets of $G$. We need to show that for any finite-to-one function $\varphi \in \omega^{\omega}$, we have $B \not \subset \bigcup_{F \in \mathscr{F}} \bigcap_{n \in \varphi(F)} K_{n} \cdot U_{n}$, where $U_{n}=\overline{\$}\left(K_{0}, \ldots, K_{n-1}\right)$ for $n \in \omega$. For every $n \in \omega$ consider the finite subset $F_{n}=f\left(K_{n}, U_{n}\right)$ of $G$. Since $\$$ is a winning strategy, $B \not \subset \bigcup_{F \in \mathscr{F}} \bigcap_{n \in \varphi(F)} F_{n} \cdot V_{n}$, where $V_{n}=\$\left(F_{0}, \ldots, F_{n-1}\right)$ for $n \in \omega$. Observe that

$$
\begin{aligned}
g\left(V_{n}\right) & =g \circ \$\left(F_{0}, \ldots, F_{n-1}\right)=g \circ \$\left(f\left(K_{0}, U_{0}\right), \ldots, f\left(K_{n-1}, U_{n-1}\right)\right) \\
& =\$\left(K_{0}, \ldots, K_{n-1}\right)=U_{n} .
\end{aligned}
$$

Then for each $F \in \mathscr{F}$, we have

$$
\begin{array}{r}
\bigcap_{n \in \varphi(F)} K_{n} \cdot U_{n} \subset \bigcap_{n \in \varphi(F)}\left(f\left(K_{n}, U_{n}\right) \cdot U_{n}\right) \cdot U_{n} \\
=\bigcap_{n \in \varphi(F)} F_{n} \cdot g\left(V_{n}\right) \cdot g\left(V_{n}\right) \subset \bigcap_{n \in \varphi(F)} F_{n} \cdot V_{n},
\end{array}
$$

and since $B \not \subset \bigcup_{F \in \mathscr{F}} \bigcap_{n \in \varphi(F)} F_{n} \cdot V_{n}$, we conclude that $B \not \subset \bigcup_{F \in \mathscr{F}} \bigcap_{n \in \varphi(F)} K_{n} \cdot U_{n}$.

Next, given a family $\mathscr{F} \subset \mathscr{P}(\omega)$ we consider the game $\mathrm{LH}_{\mathscr{F}}$ on a subset $B \subset \mathbb{R}^{\omega}$, defined as follows. Two players, I and II, choose at every step $k \in \omega$ two numbers $n_{k}$ and $m_{k}$, respectively. At the end of the game, II is declared the winner if there is a finite-to-one function $\varphi: \omega \rightarrow \omega$ such that for every $x \in B$ there is $F \in \mathscr{F}$ such that $\|x\|_{n_{k}} \leq m_{k}$ for all $k \in \varphi(F)$. 
LEMMA 3. Let $\mathscr{F} \subset \mathscr{P}(\omega)$. If player I has a winning strategy in the game $O C_{\mathscr{F}}$ on a subset $B$ of $\mathbb{R}^{\omega}$, then I has a winning strategy in the game $L H_{\mathscr{F}}$ on $B$.

PROOF. Fix a winning strategy $\$: \mathscr{K}\left(\mathbb{R}^{\omega}\right)^{<\omega} \rightarrow \mathscr{N}(0)$ of I in the game $\mathrm{OC}_{\mathscr{F}}$ on a subset $B$ of $\mathbb{R}^{\omega}$. Let $l: \mathscr{N}(0) \rightarrow \omega$ be a function assigning to each neighborhood $U \in \mathscr{N}(0)$ a number $l(U) \in \omega$ such that $\bar{B}_{l(U)}(0) \subset U$, where $\vec{B}_{n}(\varepsilon)=\left\{x \in \mathbb{R}^{\omega}\right.$ : $\left.\|x\|_{n} \leq \varepsilon\right\}$.

Define a strategy $\overline{\$}: \omega^{<\omega} \rightarrow \omega$ of $\mathrm{I}$ in the game $\mathrm{LH}_{\mathscr{F}}$ on $B$ by setting

$$
\overline{\$}\left(m_{0}, \ldots, m_{k}\right)=l \circ \$\left(\left[-m_{0}, m_{0}\right]^{\omega}, \ldots,\left[-m_{k}, m_{k}\right]^{\omega}\right)
$$

for $\left(m_{0}, \ldots, m_{k}\right) \in \omega^{<\omega}$. We claim that $\overline{\$}$ is a winning strategy for I in the game $\mathrm{LH}_{\bar{F}}$. To show this, take an arbitrary infinite sequence $\left(m_{k}\right)_{k \in \omega} \in \omega^{\omega}$ and let $n_{0}=\overline{\$}(\varnothing)$ and $n_{k}=\overline{\$}\left(m_{0}, \ldots, m_{k-1}\right)$ for $k>0$. Given a finite-to-one map $\varphi: \omega \rightarrow \omega$ we have to find a function $f \in B$ such that for any $F \in \mathscr{F}$ there is a $k \in \varphi(F)$ with $\|f\|_{n_{k}}>m_{k}$.

For each $F \in \mathscr{F}$ and $k \in \varphi(F)$, let $C_{k}=\left[-m_{k}, m_{k}\right]^{\omega} \subset \mathbb{R}^{\omega}$ and $U_{k}=$ $\$\left(C_{0}, \ldots, C_{k-1}\right)$. Observe that $l\left(U_{k}\right)=l \circ \$\left(C_{0}, \ldots, C_{k-1}\right)=n_{k}$, and thus $C_{k}+$ $\bar{B}_{l\left(U_{k}\right)}(0)=\left\{x \in \mathbb{R}^{\omega}:\|x\|_{n_{k}} \leq m_{k}\right\}$. Since $\$$ is a winning strategy for $\mathrm{I}$ in the game $\mathrm{OC}_{\mathscr{F}}$, there is $f \in B$ such that $f \notin \bigcap_{k \in \varphi(F)} C_{k}+U_{k}$ for any $F \in \mathscr{F}$. Hence there is $k \in \varphi(F)$ with $f \notin C_{k}+U_{k} \supset C_{k}+\bar{B}_{l\left(U_{k}\right)}(0)$, and it follows that $\|f\|_{n_{k}}>m_{k}$.

LEMMA 4. For any non-meager filter $\mathscr{F}$ on $\omega$ and any function $b \in \omega^{\omega}$, the first player has no winning strategy in the game $L H_{\mathscr{F}}$ on the subset $\circledast(b ; \mathscr{F})$ of $\mathbb{R}^{\omega}$.

Proof. Assume that $\$: \omega^{<\omega} \rightarrow \omega$ is a strategy of $\mathrm{I}$ in the game $\mathrm{LH}_{\mathscr{F}}$ on $\circledast(b ; \mathscr{F})$ for some non-meager filter $\mathscr{F}$ on $\omega$. To show that $\$$ is not winning we have to find an infinite number sequence $\left(m_{k}\right)_{k \in \omega} \in \omega^{\omega}$ and a finite-to-one map $\varphi: \omega \rightarrow \omega$ such that for every function $f \in \circledast(b ; \mathscr{F})$ there is $F \in \mathscr{F}$ such that $\|f\|_{n_{k}} \leq m_{k}$ for all $k \in \varphi(F)$, where $n_{k}=\$\left(m_{0}, \ldots, m_{k-1}\right)$. To construct such a sequence $\left(m_{k}\right)$, we shall use the absence of a winning strategy for I in the game $\mathfrak{G}\left(\mathfrak{F}_{r},[\omega]^{<\omega}, \mathscr{F}\right)$ (see Lemma $1(1))$. Define a strategy $\overline{\$}:\left([\omega]^{<\omega}\right)^{<\omega} \rightarrow \mathfrak{F}_{r}$ for I in the game $\mathfrak{G}\left(\mathfrak{F}_{r},[\omega]^{<\omega}, \mathscr{F}\right)$ by letting

$$
\overline{\$}\left(s_{0}, \ldots, s_{k}\right)=\left\{n \in \omega: n>\max \left\{\$\left(\|b\|_{\max s_{0}}, \ldots,\|b\|_{\max s_{k}}\right), \max s_{k}\right\}\right\}
$$

for each sequence $\left(s_{0}, \ldots, s_{k}\right)$ of finite subsets of $\omega$. Since $\overline{\$}$ is not a winning strategy for I in the game $\mathfrak{G}\left(\mathfrak{F}_{r},[\omega]^{<\omega}, \mathscr{F}\right)$, there is an infinite sequence $\left(s_{k}\right)_{k \in \omega}$ of finite subsets of $\omega$ such that $\bigcup_{k \in \omega} s_{k} \in \mathscr{F}$ and $s_{k} \subset \overline{\$}\left(s_{0}, \ldots, s_{k-1}\right)$ for all $k \in \omega$.

For every $k \in \omega$, let $m_{k}=\|b\|_{\max s_{k}}$ and $n_{k}=\$\left(m_{0}, \ldots, m_{k-1}\right)$. Then from the fact that $s_{k} \subset \overline{\$}\left(s_{0}, \ldots, s_{k-1}\right)$, we have $\min s_{k}>\max \left\{n_{k}, \max s_{k-1}\right\}$ for all $k \in \omega$. Therefore, the family $\left\{s_{k}\right\}_{k \in \omega}$ is disjoint and we can find a finite-to-one function $\varphi: \omega \rightarrow \omega$ such that $s_{k} \subset \varphi^{-1}(k)$ for every $k \in \omega$. 
Fix any function $f \in \circledast(b ; \mathscr{F})$, and choose $F \in \mathscr{F}$ such that $\|f\|_{i}<\|b\|_{i}$ for all $i \in F$. Since $\mathscr{F}$ is a filter and $\bigcup_{k \in \omega} s_{k} \in \mathscr{F}$, we can assume that $F \subset \bigcup_{k \leqslant \omega} s_{k}$. Then for any $k \in \varphi(F)$ we can find $i \in s_{k} \cap F$ and conclude that $\|f\|_{n_{k}} \leq\|f\|_{i}<$ $\|b\|_{i} \leq\|b\|_{\max s_{k}}=m_{k}$. This means that the strategy $\$$ of $\mathrm{I}$ is not winning in the game $\mathrm{LH}_{\mathscr{F}}$.

We say that a space is nowhere locally compact if none of its points has a compact neighborhood. For the proof of Theorem 4 (3) we shall need the following.

LEMMA 5. If the set $B$ is a closed nowhere locally compact subset of $\mathbb{R}^{\omega}$, then $\circledast(B ; \mathscr{F})=\mathbb{R}^{\omega}$ for every non-meager filter $\mathscr{F}$ on $\omega$.

PROOF. Assume that $\mathscr{F}$ is a non-meager filter and $B$ is a closed nowhere locally compact subset of $\mathbb{R}^{\omega}$. To show that $\circledast(B ; \mathscr{F})=\mathbb{R}^{\omega}$, fix any function $x \in \mathbb{R}^{\omega}$ and find a function $f \in \omega^{\omega}$ such that $\|x\|_{n} \leq\|f\|_{n}$ for all $n \in \omega$. For a subset $A \subset \mathbb{R}^{\omega}$ and $n \in \omega$, let $\operatorname{diam}_{n}(A)=\sup _{x, y \in A}\|x-y\|_{n}$ and $\|A\|_{n}=\sup _{a \in A}\|a\|_{n}$.

Repeating the standard inductive argument (see [14, Section 7.C]), assign to each finite number sequence $\sigma \in \omega^{<\omega}$ an open subset $U_{\sigma} \subset B$ and a number $l(\sigma) \in \omega$ so that for every $\sigma \in \omega^{<\omega}$ and $i \in \omega$ the following conditions hold:

(1) $\bar{U}_{\sigma^{\wedge} i} \subset U_{\sigma}$;

(2) $\operatorname{diam}_{l(\sigma)}\left(U_{\sigma^{\wedge} i}\right) \leq 2^{-|\sigma|}$ and $\left\|U_{\sigma^{\wedge} i}\right\|_{l(\sigma)}>i+1$;

(3) $l\left(\sigma^{\wedge} i\right)>l(\sigma)$ and $\operatorname{diam}_{l\left(\sigma^{\wedge} i\right)}\left(U_{\sigma^{\wedge} i}\right)=\infty$.

Next, define a strategy $\$:\left([\omega]^{<\omega}\right)^{<\omega} \rightarrow \mathfrak{F}_{r}$ for $I$ in the game $\mathfrak{G}\left(\mathfrak{F}_{r},[\omega]^{<\omega}, \mathscr{F}\right)$ by letting

$$
\$\left(s_{0}, \ldots, s_{k}\right)=\left\{n \in \omega: n \geq l\left(\|f\|_{\max s_{0}}, \ldots,\|f\|_{\max s_{k}}\right)\right\}
$$

for each sequence $\left(s_{0}, \ldots, s_{k}\right)$ of finite subsets of $\omega$. By Lemma $1(1), \$$ is not a winning strategy. This means that there exists a sequence $\left(s_{k}\right)_{k \in \omega}$ of finite subsets of $\omega$ such that $\bigcup_{k \in \omega} s_{k} \in \mathscr{F}$ and $s_{k} \subset \$\left(s_{0}, \ldots, s_{k-1}\right)$ for all $k \in \omega$. For every $k \in \omega$, let $m_{k}=\|f\|_{\max s_{k}}$ and $\sigma_{k}=\left(m_{0}, \ldots, m_{k}\right)$. Since $B$ is closed in the complete space $\mathbb{R}^{\omega}$, the intersection $\bigcap_{k \in \omega} U_{\sigma_{k}}$ contains a point $b \in B$. We claim that $\|f\|_{n}<\|b\|_{n}$ for all $n \in \bigcup_{k \in \omega} s_{k}$. Indeed, given $n \in \bigcup_{k \in \omega} s_{k}$, find $k \in \omega$ such that $n \in s_{k}$. Since $s_{k} \subset \$\left(s_{0}, \ldots, s_{k-1}\right)=\left[l\left(\sigma_{k-1}\right), \infty\right)$, we find $n \geq l\left(\sigma_{k-1}\right)$ and

$$
\begin{aligned}
\|b\|_{n} & \geq\|b\|_{l\left(\sigma_{k-1}\right)} \geq\left\|U_{\sigma_{k}}\right\|_{l\left(\sigma_{k-1}\right)}-\operatorname{diam}_{l\left(\sigma_{k-1}\right)}\left(U_{\sigma_{k}}\right) \\
& >m_{k}+1-2^{-\left|\sigma_{k-1}\right|} \geq m_{k}=\|f\|_{\max s_{k}} \geq\|\dot{f}\|_{n} .
\end{aligned}
$$

Thus $\|f\|_{n}<\|b\|_{n}$ for all $n \in \bigcup_{k \in \omega} s_{k}$, as claimed. Finally, since we have $\|x\|_{n} \leq$ $\|f\|_{n}$ for all $n \in \omega$, it follows that $x \in \circledast(B ; \mathscr{F})$, as required.

We now prove another difficult lemma, which will be used in the proof of statements (3) and (4) of Theorem 5. 
LEMMa 6. Assume that $\mathscr{F} \subset \mathscr{F}^{\prime}$ are two filters on $\omega$ such that $I$ has no winning strategy in the game $\mathfrak{G}\left(\mathscr{F},[\omega]^{<\omega}, \mathscr{F}^{\prime}\right)$. Then for any subset $A \subset \circledast(\mathscr{F})$, the closure $\bar{A}$ of $A$ in $\circledast\left(\mathscr{F}^{\prime}\right)$ is a Baire space.

PROOF. We have to verify that the intersection $\bigcap_{k \in \mathbb{N}} U_{k}$ of any decreasing sequence $\left(U_{k}\right)_{k \in \mathbb{N}}$ of dense open subsets of $\bar{A}$ is dense in $\bar{A}$. It suffices to verify that for any non-empty open subset $U_{0} \subset \bar{A}$, the intersection $\bigcap_{k \in \omega} U_{k}$ is non-empty.

Fix any function $h_{\emptyset} \in U_{0} \cap A$. Since $U_{0}$ is a neighborhood of $h_{\emptyset}$ in $\bar{A}$, there are $l(\emptyset) \in \omega$ and $\varepsilon(\emptyset)>0$ such that $x \in U_{0}$ for any function $x \in \bar{A}$ satisfying $\left\|x-h_{\emptyset}\right\|_{l(\emptyset)} \leq \varepsilon(\emptyset)$. Since $h_{\emptyset} \in A \subset \circledast(\mathscr{F})$, we can find a subset $F(\emptyset) \in \mathscr{F}$ such that $F(\emptyset) \subset[l(\emptyset)+1, \infty)$ and $\left\|h_{\emptyset}\right\|_{n}<n$ for all $n \in F(\emptyset)$.

By induction over the tree $\omega^{<\omega}$, assign to every finite sequence $\sigma \in \omega^{<\omega}$ a function $h_{\sigma} \in A$, a set $F(\sigma) \in \mathscr{F}$, a number $l(\sigma) \in \omega$, and real positive numbers $\varepsilon(\sigma), \delta(\sigma)$ such that the following conditions hold for every $\sigma \in \omega^{<\omega}$ and $i \in \omega$ :

(1) $\delta\left(\sigma^{\wedge} i\right) \leq \delta(\sigma)$ and $\delta\left(\sigma^{\wedge} i\right) \leq \frac{1}{2} \min _{j \in F(\sigma) \cap[0, i]}\left(j-\left\|h_{\sigma}\right\|_{j}\right)$;

(2) $h_{\sigma^{\wedge} i} \in A \cap U_{\left|\sigma^{\wedge} i\right|}$ and $\left\|h_{\sigma^{\wedge} i}-h_{\sigma}\right\|_{i} \leq 2^{-\left|\sigma^{\wedge} i\right|} \min \left\{\varepsilon(\sigma), \delta\left(\sigma^{\wedge} i\right)\right\}$;

(3) $\varepsilon\left(\sigma^{\wedge} i\right) \leq \varepsilon(\sigma)$;

(4) for all $x \in \bar{A},\left\|x-h_{\sigma^{\wedge} i}\right\|_{l\left(\sigma^{\wedge} i\right)} \leq \varepsilon\left(\sigma^{\wedge} i\right)$ implies $x \in U_{\left|\sigma^{\wedge} i\right|}$;

(5) $\left\|h_{\sigma^{\wedge} i}\right\|_{j}<j$ for all $j \in F\left(\sigma^{\wedge} i\right)$;

(6) $\max \left\{i, l\left(\sigma^{\wedge} i\right)\right\}<\min F\left(\sigma^{\wedge} i\right)$.

Define a strategy $\$:\left([\omega]^{<\omega}\right)^{<\omega} \rightarrow \mathscr{F}$ for I in the game $\mathfrak{G}\left(\mathscr{F},[\omega]^{<\omega}, \mathscr{F}^{\prime}\right)$ letting $\$\left(s_{0}, \ldots, s_{k}\right)=F\left(\max s_{0}, \ldots, \max s_{k}\right)$ for $\left(s_{0}, \ldots, s_{k}\right) \in\left([\omega]^{<\omega}\right)^{<\omega}$. According to our hypothesis, $\$$ is not a winning strategy of I. Hence there is a sequence $\left(s_{k}\right)_{k \in \omega}$ of finite subsets of $\omega$ such that $\bigcup_{k \in \omega} s_{k} \in \mathscr{F}^{\prime}$ and $s_{k} \subset \$\left(s_{0}, \ldots, s_{k-1}\right)$ for all $k \in \omega$.

For every $k \in \omega$, let $m_{k}=\max s_{k}$ and $\sigma_{k}=\left(m_{0}, \ldots, m_{k}\right)$. Consider the function sequence $\left(h_{\sigma_{k}}\right)_{k \in \omega} \subset A$. The condition (2) implies that this sequence is Cauchy in $\mathbb{B}^{\omega}$, and thus has a limit $h_{\infty} \in \mathbb{R}^{\omega}$. Let us show that $\left\|h_{\infty}\right\|_{i}<i$ for all $i \in \bigcup_{k \in \omega} s_{k}$. Given arbitrary $i \in F^{\prime}=\bigcup_{k \in \omega} s_{k}$, find $k \in \omega$ with $i \in s_{k}$. Since $i \in s_{k} \subset \$\left(s_{0}, \ldots, s_{k-1}\right)=$ $F\left(\sigma_{k-1}\right)$ and $i \leq \max s_{k}=m_{k}$, we have

$$
\begin{aligned}
\left\|h_{\infty}\right\|_{i} & \leq\left\|h_{\sigma_{k-1}}\right\|_{i}+\left\|h_{\infty}-h_{\sigma_{k-1}}\right\|_{i} \leq\left\|h_{\sigma_{k-1}}\right\|_{i}+\sum_{p=k}^{\infty}\left\|h_{\sigma_{p}}-h_{\sigma_{p-1}}\right\|_{m_{p}} \\
& \leq\left\|h_{\sigma_{k-1}}\right\|_{i}+\sum_{p=k}^{\infty} \frac{1}{2^{\left|\sigma_{p}\right|}} \delta\left(\sigma_{p}\right) \leq\left\|h_{\sigma_{k-1}}\right\|_{i}+\sum_{p=k}^{\infty} \frac{1}{2^{p+1}} \delta\left(\sigma_{k}\right) \\
& \leq\left\|h_{\sigma_{k-1}}\right\|_{i}+\delta\left(\sigma_{k}\right) \leq\left\|h_{\sigma_{k-1}}\right\|_{i}+\frac{1}{2}\left(i-\left\|h_{\sigma_{k-1}}\right\|_{i}\right)=\frac{1}{2}\left(i+\left\|h_{\sigma_{k-1}}\right\|_{i}\right)<i,
\end{aligned}
$$

and thus $h_{\infty} \in \circledast\left(\mathscr{F}^{\prime}\right)$. Since $h_{\infty}$ is the limit of the sequence $\left(h_{\sigma_{k}}\right)_{k \in \omega} \subset A$, we have $h_{\infty} \in \bar{A}$. 
Now it remains to verify that $h_{\infty} \in U_{k}=U_{\left|\sigma_{k-1}\right|}$ for all $k \in \omega$. By (4), it suffices to show that $\left\|h_{\infty}-h_{\sigma_{k-1}}\right\|_{l\left(\sigma_{k-1}\right)} \leq \varepsilon\left(\sigma_{k-1}\right)$. But using the fact that $l\left(\sigma_{k-1}\right)<$ $\min F\left(\sigma_{k-1}\right) \leq \min s_{k} \leq m_{k}$, we find

$$
\begin{aligned}
\left\|h_{\infty}-h_{\sigma_{k-1}}\right\|_{l\left(\sigma_{k-1}\right)} & \leq \sum_{p=k}^{\infty}\left\|h_{\sigma_{p}}-h_{\sigma_{p-1}}\right\|_{l\left(\sigma_{k-1}\right)} \leq \sum_{p=k}^{\infty}\left\|h_{\sigma_{p}}-h_{\sigma_{p-1}}\right\|_{m_{p}} \\
& \leq \sum_{p=k}^{\infty} \frac{1}{2^{\left|\sigma_{p}\right|}} \varepsilon\left(\sigma_{p-1}\right) \leq \sum_{p=k}^{\infty} \frac{1}{2^{p+1}} \varepsilon\left(\sigma_{k-1}\right) \leq \varepsilon\left(\sigma_{k-1}\right),
\end{aligned}
$$

and the proof is complete.

In our subsequent considerations, we shall often need the following simple but useful result.

LEMMA 7. For any $\sigma$-bounded subset $B \subset \mathbb{R}^{\omega}$, there is an increasing function $b \in \omega^{\omega}$ such that $\circledast(B ; \mathscr{F}) \subset \circledast(b ; \mathscr{F})$ for every semi-filter $\mathscr{F}$.

PROOF. Write $B=\bigcup_{n \in \omega} B_{n}$, where $\left(B_{n}\right)_{n \in \omega}$ is an increasing sequence of bounded subsets of $\mathbb{R}^{\omega}$. Let $b(-1)=0$, and for every $n \in \omega$ fix by induction any $b(n)>$ $b(n-1)$ with $b(n) \geq \sup \left\{\|x\|_{n}: x \in B_{n}\right\}$. Clearly, $b$ is validly defined. To prove that $\circledast(B ; \mathscr{F}) \subset \circledast(b ; \mathscr{F})$ for any semi-filter $\mathscr{F}$, fix any function $x \in \circledast(B ; \mathscr{F})$ and, by the definition of $\circledast(B ; \mathscr{F})$, find a function $y \in B$ and a subset $F \in \mathscr{F}$ such that $\|x\|_{i}<\|y\|_{i}$ for all $i \in F$. Next, find $n \in \mathbb{N}$ such that $y \in B_{n}$ and let $F^{\prime}=F \cap[n, \infty) \in \mathscr{F}$. Then $\|x\|_{i}<\|y\|_{i} \leq b(i)=\|b\|_{i}$ for all $i \in F^{\prime}$, and thus $x \in \circledast(b ; \mathscr{F})$.

In the sequel we shall need a characterization of meager semi-filters which generalizes the Talagrand characterization of meager filters [21] and can be proved by the same argument (see [24, page 32]).

PROPOSITION 3. For a semi-filter $\mathscr{F}$, the following conditions are equivalent:

(1) $\mathscr{F}$ is meager in $\mathscr{P}(\omega)$;

(2) $\mathscr{F}$ lies in a $\sigma$-compact subset of $[\omega]^{\omega}$;

(3) there is an increasing number sequence $\left(m_{i}\right)$ such that each $F \in \mathscr{F}$ meets all but finitely many intervals $\left[m_{i}, m_{i+1}\right)$.

\section{Proofs of main results}

Proof OF THEOREM 2. (1) First, assume that the set $B$ is $o_{\mathscr{F}}$-bounded in $\mathbb{R}^{\omega}$. For every $n \in \omega$ consider the open neighborhood $U_{n}=\left\{x \in \mathbb{R}^{\omega}:\|x\|_{n}<1\right\}$ of the origin 
of $\mathbb{R}^{\omega}$. Since the set $B$ is $o_{\mathcal{F}}$-bounded in $\mathbb{R}^{\omega}$ there is a sequence $\left(F_{n}\right)_{n \in \omega}$ of finite subsets of $\mathbb{R}^{\omega}$ and a finite-to-one function $\varphi \in \omega^{\omega}$ such that $B \subset \bigcup_{F \in \mathscr{F}} \bigcap_{n \in \varphi(F)} F_{n}+U_{n}$. We claim that $B \subset \circledast(b ; \varphi[\mathscr{F}])$, where $b(n)=1+\max \left\{\|x\|_{n}: x \in F_{n}\right\}, n \in \omega$.

Take any $x \in B$, and find $F \in \mathscr{F}$ such that for every $n \in \varphi(F)$ we have $x \in F_{n}+U_{n}$. Then $\|x\|_{n}<b(n)$, and hence $x \in \circledast(b ; \varphi[\mathscr{F}])$.

Next, assuming that $B \subset \circledast(b, \varphi[\mathscr{F}])$ for some function $b \in \mathbb{R}^{\omega}$ and some finiteto-one function $\varphi \in \omega^{\omega}$, we shall show that the set $B$ is $o_{\mathscr{F}}$-bounded in $\mathbb{R}^{\omega}$. Let $\left(U_{k}\right)_{k \in \omega}$ be a sequence of neighborhoods of the origin of $\mathbb{R}^{\omega}$. For every $k \in \omega$, find $n_{k} \in \omega$ and $\varepsilon_{k}>0$ such that $\bar{B}_{n_{k}}\left(\varepsilon_{k}\right) \subset U_{k}$, where $\bar{B}_{n}(\varepsilon)=\left\{x \in \mathbb{R}^{\omega}:\|x\|_{n} \leq \varepsilon\right\}$. We can assume that $n_{k+1}>n_{k}$ for all $k \in \omega$. Now for every $k \in \omega$ find a finite subset $F_{k} \subset \mathbb{R}^{\omega}$ such that $\left[-\uparrow b\left(n_{k+1}\right), \uparrow b\left(n_{k+1}\right)\right]^{\omega} \subset F_{k}+\bar{B}_{n_{k}}\left(\varepsilon_{k}\right)$. Let $\psi \in \omega^{\omega}$ be the finite-to-one function defined by $\psi^{-1}(k)=\left[n_{k}, n_{k+1}\right)$ for $k \in \omega$.

We claim that $B \subset \bigcup_{F \in \mathscr{F}} \bigcap_{k \in \psi \circ \varphi(F)} F_{k}+U_{k}$. Take any $x \in B \subset \circledast(b ; \varphi[\mathscr{F}])$ and find $F \in \mathscr{F}$ such that $\uparrow x(i)<\uparrow b(i)$ for all $i \in \varphi(F)$. Since $\mathscr{F}$ is a semi-filter and $\varphi$ is finite-to-one, we can assume that $\min \varphi(F) \geq n_{0}$. In this case, for every $k \in \psi \circ \varphi(F)$ we can find a number $i \in\left[n_{k}, n_{k+1}\right) \cap \varphi(F)$. Then

$$
\begin{aligned}
x & \in[-\uparrow b(i), \uparrow b(i)]^{\omega}+\bar{B}_{i}(0) \subset\left[-\uparrow b\left(n_{k+1}\right), \uparrow b\left(n_{k+1}\right)\right]^{\omega}+\bar{B}_{n_{k}}(0) \\
& \subset F_{k}+\bar{B}_{n_{k}}\left(\varepsilon_{k}\right)+\bar{B}_{n_{k}}(0)=F_{k}+\bar{B}_{n_{k}}\left(\varepsilon_{k}\right) \subset F_{k}+U_{k} .
\end{aligned}
$$

Hence $B \subset \bigcup_{F \in \mathscr{F}} \bigcap_{k \in \psi(\varphi(F))} F_{k}+U_{k}$, and so $B$ is $o_{\mathscr{F}}$-bounded.

(2) In light of the previous item, to prove the second statement of Theorem 2, it suffices to verify that each $o$-bounded subset $B \subset \mathbb{R}^{\omega}$ is $o_{[\omega]^{\omega}}$-bounded. Fix a sequence $\left(U_{n}\right)_{n \in \omega}$ of neighborhoods of the origin of $\mathbb{R}^{\omega}$. Since the set $B$ is $o$-bounded in $\mathbb{R}^{\omega}$, for every $k \in \omega$ there is a sequence $\left(F_{n}^{k}\right)_{n \geq k}$ of finite subsets of $\mathbb{R}^{\omega}$ such that $B \subset \bigcup_{n \geq k} F_{n}^{k}+U_{n}$. For every $k \in \omega$, let $F_{n}=\bigcup_{k \leq n} F_{n}^{k}$, and note that $B \subset \bigcup_{n \geq k} F_{n}^{k}+U_{n} \subset \bigcup_{n \geq k} F_{n}+U_{n}$. Then for every $x \in B$ there is an infinite subset $S \subset \omega$ such that $x \in F_{n}+U_{n}$ for each $n \in S$. Hence $B \subset \bigcup_{S \in[\omega]^{\omega}} \bigcap_{n \in S} F_{n}+U_{n}$, which means that the set $B$ is $o_{[\omega]}{ }^{\omega}$-bounded.

Proof of THEOREM 4. Let $B \subset \mathbb{R}^{\omega}$ and $\mathscr{F}$ be a semi-filter on $\omega$. It will be convenient to start with the last assertion of the theorem.

(4) We must show that $\circledast(B ; \mathscr{F})$ is $\sigma$-bounded if and only if either $\sup _{n \in \omega}\|b\|_{n}<\infty$ for all $b \in B$ or $B$ is $\sigma$-bounded and $\mathscr{F}$ is meager.

If $\sup _{n \in \omega}\|b\|_{n}<\infty$ for all $b \in B$, then the set $\circledast(b, \mathscr{F})$ consists of bounded functions and hence is $\sigma$-bounded in $\mathbb{R}^{\omega}$. Next, assume that the set $B$ is $\sigma$-bounded and the semi-filter $\mathscr{F}$ is meager. By Lemma $7, \circledast(B ; \mathscr{F}) \subset \circledast(b ; \mathscr{F})$ for some increasing function $b: \omega \rightarrow \omega$. Using Proposition 3, find an increasing number sequence $\left(m_{i}\right)$ such that each element $F \in \mathscr{F}$ meets almost all half-intervals $\left[m_{i}, m_{i+1}\right)$. Consider the increasing function $f: \omega \rightarrow \omega$ defined by $f(i)=b\left(m_{i+2}\right)$ for $i \in \omega$. The $\sigma$-boundedness of the set $\circledast(b ; \mathscr{F})$ will follow as soon as we show that $\uparrow x \leq^{*} f$ 
for every $x \in \circledast(b ; \mathscr{F})$. Indeed, given any $x \in \circledast(b ; \mathscr{F})$, find $F \in \mathscr{F}$ such that $\|x\|_{i}<b(i)$ for all $i \in F$. Next, find $i_{0} \in \omega$ such that $F \cap\left[m_{i}, m_{i+1}\right) \neq \emptyset$ for all $i \geq i_{0}$. Then for any $k \geq m_{i_{0}}$ there is a number $i \geq i_{0}$ with $k \in\left[m_{i}, m_{i+1}\right)$ and an element $n \in F \cap\left[m_{i+1}, m_{i+2}\right)$. Observing that

$$
\|x\|_{k} \leq\|x\|_{n}<b(n) \leq b\left(m_{i+2}\right)=f(i) \leq f\left(m_{i}\right) \leq f(k),
$$

we conclude that $\uparrow x \leq * f$.

Now assume that the set $\circledast(B ; \mathscr{F})$ is $\sigma$-bounded and $\sup _{n \in \omega}\|b\|_{n}=\infty$ for some $b \in B$. We have to show that the set $B$ is $\sigma$-bounded and $\mathscr{F}$ is meager. Using the $\sigma$-boundedness of the set $\circledast(B ; \mathscr{F})$, find an increasing function $f: \omega \rightarrow \omega$ such that $\uparrow x \leq^{*} f$ for any $x \in \circledast(B ; \mathscr{F})$. Let $m_{0}=0$, and by recursion define an increasing number sequence $\left(m_{i}\right)$ by setting $m_{i+1}=\min \left\{k \in \omega:\|b\|_{k}>2 f\left(m_{i}\right)\right\}$. We claim that each element $F \in \mathscr{F}$ meets almost all half-intervals $\left[m_{i}, m_{i+1}\right)$. Replacing $F$ by $F \cap[n, \infty)$ for sufficiently large $n$, if necessary, we can assume that $\|b\|_{i}>0$ for all $i \in F$. Then the function $b_{F} / 2$, where $b_{F}(i)=\|b\|_{\min F \cap[i, \infty)}$, belongs to $\circledast(B ; \mathscr{F})$, and thus $b_{F} / 2 \leq{ }^{*} f$. Find $i_{0} \in \omega$ such that $b_{F}(k) \leq 2 f(k)$ for all $k \geq m_{i_{0}}$. We claim that for every $i \geq i_{0}$ the set $F$ meets the half-interval $\left[m_{i}, m_{i+1}\right)$. Indeed, assuming that $F \cap\left[m_{i}, m_{i+1}\right)=\emptyset$ for some $i \geq i_{0}$ we would get $b\left(m_{i+1}\right) \leq b_{F}\left(m_{i}\right) \leq 2 f\left(m_{i}\right)$, which contradicts the definition of $m_{i+1}$. Therefore $F$ meets all the half-intervals $\left[m_{i}, m_{i+1}\right)$ for $i \geq i_{0}$, and by Proposition 3, the semi-filter $\mathscr{F}$ is meager.

It remains to show that the set $B$ is $\sigma$-bounded. Observe that for any $b \in B$ the function $b / 2$ belongs to $\circledast(b, \mathscr{F})$, and thus $b / 2 \leq^{*} f$. This is equivalent to saying that $b \leq^{*} 2 f$ for each $b \in B$, and we conclude that $B$ is $\sigma$-bounded in $\mathbb{R}^{\omega}$.

Now we are able to prove the first item of Theorem 4 .

(1) If the set $B$ is $\sigma$-bounded in $\mathbb{R}^{\omega}$, then we can apply Lemma 7 to find an increasing function $b \in \omega^{\omega}$ with $\circledast(B ; \mathscr{F}) \subset \circledast(b ; \mathscr{F})$. Applying Theorem 2 , we conclude that the set $\circledast(b ; \mathscr{F})$, and therefore the set $\circledast(B ; \mathscr{F})$, is $o_{\mathscr{F}}$-bounded.

Now we show that $\circledast(b ; \mathscr{F})$ is $\boldsymbol{I}_{\mathscr{F}}$-bounded if $\mathscr{F}$ is a filter. If the filter $\mathscr{F}$ is meager, then $\circledast(b ; \mathscr{F})$ is $\sigma$-bounded according to item (4). Consequently, the set $\circledast(B ; \mathscr{F}) \subset \circledast(b ; \mathscr{F})$ is $\sigma$-bounded and hence is $I_{\mathscr{F}}$-bounded. If the filter $\mathscr{F}$ is non-meager, then Lemmas $2-4$ imply that the first player has no winning strategy in the games $\mathrm{LH}_{\mathscr{F}}, \mathrm{OC}_{\mathscr{F}}$ and $\mathrm{OF}_{\mathscr{F}}$ on $\circledast(b ; \mathscr{F})$. This implies that the set $\circledast(b ; \mathscr{F})$, and hence the set $\circledast(B ; \mathscr{F})$, is $I_{\mathscr{F}}$-bounded.

(2) To prove the second item of Theorem 4 it suffices to verify that a subset $B \subset \mathbb{R}^{\omega}$ is $o$-bounded provided $\circledast\left(B ; \mathfrak{F}_{r}\right) \neq \mathbb{R}^{\omega}$. Fix any function $f \in \mathbb{R}^{\omega} \backslash \circledast\left(B ; \mathfrak{F}_{r}\right)$. We claim that $B \subset \circledast\left(|f|+1 ;[\omega]^{\omega}\right)$. Indeed, let $b \in B$. Then for each cofinite set $F \subset \omega$ there exists $n \in F$ such that $\|f\|_{n} \geq\|b\|_{n}$, and it follows that there exists an infinite set $F^{\prime} \subset \omega$ such that $\|f\|_{n} \geq\|b\|_{n}$ for all $n \in F^{\prime}$. This implies immediately that $b \in \circledast\left(|f|+1 ;[\omega]^{\omega}\right)$, and so we have $B \subset \circledast\left(|f|+1 ;[\omega]^{\omega}\right)$, as claimed. It follows from this, by Theorem 2, that the set $B$ is $o$-bounded. 
(3) Assume that $B$ is an analytic subspace of $\mathbb{R}^{\omega}$ and $\mathscr{F}$ is a non-meager filter. If the set $B$ is $\sigma$-bounded, then by item $(1)$, the set $\circledast(B ; \mathscr{F})$ is $o_{\mathscr{F}}$-bounded in $\mathbb{R}^{\omega}$, and thus $\circledast(B ; \mathscr{F}) \neq \mathbb{R}^{\omega}$. If $B$ is not $\sigma$-bounded, then $B$ contains a subset $B^{\prime} \subset B$ which is nowhere locally compact and is closed in $\mathbb{R}^{\omega}$ (see [14, Corollary 21.23]). In this situation we can apply Lemma 5 , and conclude that

$$
\mathbb{R}^{\omega}=\circledast\left(B^{\prime} ; \mathscr{F}\right) \subset \circledast(B ; \mathscr{F})=\mathbb{R}^{\omega},
$$

and that in particular $\circledast(B ; \mathscr{F})$ is not $o_{\mathscr{F}}$-bounded.

PROOF OF THEOREM 5. Let $B \subset \mathbb{R}^{\omega}$ and $\mathscr{F}$ be a semi-filter on $\omega$.

(1) Recall that for $b \in \mathbb{R}^{\omega}$ and $F \in[\omega]^{\omega}$, we denote by $b_{F}$ the function in $\mathbb{R}^{\omega}$ defined by $b_{F}(i)=\|b\|_{\min (F \cap[i, \infty))}$ for all $i \in \omega$. It is easy to check that the mapping $\mathbb{R}^{\omega} \times$ $[\omega]^{\omega} \rightarrow \mathbb{R}^{\omega}$ defined by $(b, F) \mapsto b_{F}$ is continuous, and it then follows immediately that the map $\Psi: \mathbb{R}^{\omega} \times[\omega]^{\omega} \times(-1,1)^{\omega} \rightarrow \mathbb{R}^{\omega}$ defined by $\Psi:(b, F, t) \mapsto t \cdot b_{F}$ is also continuous, where $t \cdot b_{F}$ is the coordinatewise product of the functions $t$ and $b_{F}$. Clearly, the space $\circledast(B ; \mathscr{F})$ is the image of the product $B \times \mathscr{F} \times(-1,1)^{\omega}$ under $\Psi$.

(2) If $B, \mathscr{F} \in \Sigma_{n}^{1}$ for some $n \in \mathbb{N}$, then $B \times \mathscr{F} \times(-1,1)^{\omega} \in \Sigma_{n}^{1}$ (see [14, Proposition 37.1]), and $\circledast(B ; \mathscr{F})$, being a continuous image of $B \times \mathscr{F} \times(-1,1)^{\omega}$, also belongs to the class $\Sigma_{n}^{1}$.

(3) If $\mathscr{F}$ is a non-meager filter, then by Lemma 1 (1) the first player has no winning strategy in the game $\mathfrak{G}\left(\mathfrak{F}_{r},[\omega]^{<w}, \mathscr{F}\right)$. Applying Lemma 6 , we conclude that the closure $\bar{A}$ of any subset $A \subset \circledast\left(\mathfrak{F}_{r}\right)$ in $\circledast(\mathscr{F})$ is a Baire space. In particular, the space $\circledast(\mathscr{F})$, being the closure of $\circledast\left(\mathfrak{F}_{r}\right)$, is Baire.

(4) If $\mathscr{F}$ is a non-meager $P$-filter, then by Lemma 1 (2), the first player has no winning strategy in the game $\mathfrak{G}\left(\mathscr{F},[\omega]^{<\omega}, \mathscr{F}\right)$. Applying Lemma 6 , we conclude that the closure of any subset $A \subset \circledast(\mathscr{F})$ in $\circledast(\mathscr{F})$ is a Baire space. The space $\circledast(\mathscr{F})$ is thus hereditarily Baire.

(5) Suppose that $\left(b_{k}\right)_{k \in \omega} \subset B$ is a sequence of unbounded functions such that $\uparrow b_{k}<{ }^{*} \uparrow b_{k+1}$ for all $k \in \omega$ and such that for every $b \in B$ there is $k \in \omega$ such that $\uparrow b \leq^{*} \uparrow b_{k}$. Let $l_{0}=0$. By induction, construct an increasing number sequence $\left(l_{k}\right)_{k \in \omega} \in \omega^{\omega}$ such that for every $k \in \omega$ and every $i \geq l_{k}$ we have $\uparrow b_{k-1}(i)<\uparrow b_{k}(i)$ (which is equivalent to $\left\|b_{k-1}\right\|_{i}<\left\|b_{k}\right\|_{i}$ ). It follows that for every $k<n$ and every $i \geq l_{n}$ we have $\left\|b_{k}\right\|_{i}<\left\|b_{n}\right\|_{i}$.

Given a subset $A$ of $\omega$, consider the function $f_{A} \in \mathbb{R}^{\omega}$ defined for $i \in \omega$ by $f_{A}(i)=$ $\left\|b_{k}\right\|_{i}$, where $k \in \omega$ is chosen to satisfy the condition $\max ([0, i] \cap(A \cup\{0\})) \in\left[l_{k}, l_{k+1}\right)$. We claim that $f_{A} \in \circledast(B ; \mathscr{F})$ if and only if the set $A$ is finite.

Assuming that $A$ is finite, find $k \in \omega$ such that $\max (A \cup\{0\}) \in\left[l_{k}, l_{k+1}\right)$, and observe that $\left|f_{A}(i)\right|=\left\|b_{k}\right\|_{i}<\left\|b_{k+1}\right\|_{i}$ for all $i \geq l_{k+1}$. This yields $f_{A} \in \circledast(B ; \mathscr{F})$.

Next, assume that the set $A$ is infinite. To show that $f_{A} \notin \circledast(B ; \mathscr{F})$ it suffices for every $b \in B$ to find $m \in \omega$ such that $\left\|f_{A}\right\|_{i} \geq\|b\|_{i}$ for all $i \geq m$. Given 
arbitrary $b \in B$, find $p \in \omega$ such that $\uparrow b \leq^{*} \uparrow b_{p}$. Next, find $q \geq p$ such that $A \cap\left[l_{q}, l_{q+1}\right) \neq \emptyset$ and $\uparrow b(i) \leq \uparrow b_{p}(i)$ for all $i \geq l_{q}$. Given arbitrary $i \geq l_{q+1}$, find $k \in \omega$ such that $\max \left([0, i] \cap(A \cup\{0\}) \in\left[l_{k}, l_{k+1}\right)\right.$, and observe that $k \geq q \geq p$ and $\left\|f_{A}\right\|_{i} \geq\left|f_{A}(i)\right|=\left\|b_{k}\right\|_{i} \geq\left\|b_{p}\right\|_{i} \geq\|b\|_{i}$.

Now consider the map $\Psi: \mathscr{P}(\omega) \rightarrow \mathbb{B}^{\omega}$ assigning to each subset $A \subset \omega$ the function $f_{A}$. It is easy to see that the map $\Psi$ is continuous. We have already proved that $\Psi(\mathscr{P}(\omega)) \cap \circledast(B ; \mathscr{F})=\Psi\left([\omega]^{<\omega}\right)$, and thus $\Psi\left([\omega]^{<\omega}\right)$ is a countable closed subset of $\circledast(B ; \mathscr{F})$ because the set $\Psi(\mathscr{P}(\omega))$ is compact. It remains to observe that the space $\Psi\left([\omega]^{<\omega}\right)$ has no isolated points and that $\Psi\left([\omega]^{<\omega}\right)$ is thus a closed meager subspace of $\circledast(B ; \mathscr{F})$. This implies that the space $\circledast(B ; \mathscr{F})$ is not hereditarily Baire.

Proof of Theorem 6. The implications (1) implies (5), (1) implies (4), (4) implies (3), and (3) implies (2) are trivial.

Though the implication (2) implies (1) follows from Theorem 1 (1) we give a short proof to make the paper self-contained. So, suppose that $B$ is a II-bounded subset of $\mathbb{R}^{\omega}$ and let $\$: \mathscr{N}(0)^{<\omega} \rightarrow\left[\mathbb{R}^{\omega}\right]^{<\omega}$ be a winning strategy of the second player in the game OF on the set $B$. For every $n \in \omega$, let $U_{n}=\left\{x \in \mathbb{R}^{\omega}:\|x\|_{n}<1\right\}$.

Our crucial observation is that

$$
b \in \bigcup_{\left(n_{1}, \ldots, n_{k}\right) \in \omega^{<\omega}} \bigcap_{m \in \omega} \$\left(U_{n_{1}}, \ldots, U_{n_{k}}, U_{m}\right)+U_{m}
$$

for every $b \in B$. Indeed, assuming that this is false, we can construct by induction an infinite sequence $\left(n_{k}\right)_{k \in \omega} \in \omega^{\omega}$ such that $b \notin \$\left(U_{n_{1}}, \ldots, U_{n_{k}}\right)+U_{n_{k}}$ for every $k \in \omega$. Then $b \notin \bigcup_{k \in \omega} \$\left(U_{n_{1}}, \ldots, U_{n_{k}}\right)+U_{n_{k}}$, which shows that the strategy $\$$ is not winning, a contradiction. Hence $B$ is contained in the set

$$
\bigcup_{\left(n_{1}, \ldots, n_{k}\right) \in \omega^{<\omega}} \bigcap_{m \in \omega} \$\left(U_{n_{1}}, \ldots, U_{n_{k}}, U_{m}\right)+U_{m},
$$

which is the countable union of the bounded subsets $\bigcap_{m \in \omega} \$\left(U_{n_{1}}, \ldots, U_{n_{k}}, U_{m}\right)+U_{m}$ of $\mathbb{R}^{\omega}$ for $\left(n_{1}, \ldots, n_{k}\right) \in \omega^{<\omega}$.

(5) implies (1): Assume that a set $B \subset \mathbb{R}^{\omega}$ is $o_{\mathscr{F}}$-bounded for some meager semifilter $\mathscr{F}$. By Theorem $2, B \subset \circledast(b ; \varphi[\mathscr{F}])$ for some $b \in \omega^{\omega}$ and some finite-to-one function $\varphi \in \omega^{\omega}$. The $\sigma$-boundedness of the set $\circledast(b ; \varphi[\mathscr{F}])$ will therefore follow from Theorem 4 (4) as soon as we prove that the semi-filter $\varphi[\mathscr{F}]$ is meager.

By Proposition 3, the semi-filter $\mathscr{F}$, being meager, lies in a $\sigma$-compact subset $\mathscr{K} \subset[\omega]^{\omega}$. Consider the map $\mathscr{P}(\varphi): \mathscr{P}(\omega) \rightarrow \mathscr{P}(\omega)$ defined by $\mathscr{P}(\varphi)(A)=\varphi(A)$ for $A \in \mathscr{P}(\omega)$. Since $\varphi$ is finite-to-one, $\mathscr{P}(\varphi)\left([\omega]^{\omega}\right) \subset[\omega]^{\omega}$. The continuity of $\mathscr{P}(\varphi)$ implies that the set $\mathscr{L}=\{\varphi(F): F \in \mathscr{F}\}$ is $\sigma$-compact in $[\omega]^{\omega}$. Finally, observe that

$$
\varphi[\mathscr{F}] \subset \uparrow \mathscr{L}=\{E \subset \omega: \exists L \in \mathscr{L} \text { with } L \subset E\}
$$


and that the set $\uparrow \mathscr{L}$ is $\sigma$-compact in $[\omega]^{\omega}$. Applying Proposition 3 to the semi-filter $\varphi[\mathscr{F}]$, we conclude that it is meager.

PROOF OF THEOREM 7. (1) Suppose that $B \subset \mathbb{R}^{\omega}$ is $o_{\mathscr{F}}$-bounded for some filter $\mathscr{F}$. Applying Theorem 2, find a function $b \in \omega^{\omega}$ and a finite-to-one function $\varphi: \omega \rightarrow \omega$ such that $B \subset \circledast(b ; \varphi[\mathscr{F}])$. Since $\varphi[\mathscr{F}]$ is a filter, we can apply Theorem 4 (1) to conclude that the set $\circledast(b ; \varphi[\mathscr{F}])$ is $I_{\varphi(\mathscr{F}]}$-bounded and hence $I_{\mathscr{F}}$-bounded, and that the subset $B \subset \circledast(b ; \varphi[\mathscr{F}])$ is therefore also $I_{\mathscr{F}}$-bounded.

(2) Suppose that $\mathscr{F}_{1}, \ldots, \mathscr{F}_{n}$ are filters and $b \in \omega^{\omega}$. According to Lemmas 2-3, to prove that the union $U=\circledast\left(b ; \mathscr{F}_{1}\right) \cup \cdots \cup \circledast\left(b ; \mathscr{F}_{n}\right)$ is $I_{[\omega]}$-bounded, it suffices to verify that the first player has no winning strategy in the game $\mathrm{LH}_{[\omega]^{\omega}}$ on $U$. To show this we shall use the argument from the proof of Theorem 2.12 of [17]. Suppose that the first player has a winning strategy $\$: \omega^{<\omega} \rightarrow \omega$ in the game $\operatorname{LH}_{[\omega]^{\omega}}$ on $U$. Without loss of generality, we can assume that $n \geq 2$, that the function $b$ is increasing and takes positive integer values, and that the strategy $\$$ is monotone in the sense that $\$\left(m_{0}, \ldots, m_{k}\right)>m_{k}$ for any finite sequence $\left(m_{0}, \ldots, m_{k}\right) \in \omega^{<\omega}$. To beat the strategy $\$$ of $I$, the second player will simultaneously play $(3 n+1)$ games, and will win in one of these games.

For every $p \in\{0, \ldots, 3 n\}$, define a number sequence $\left(m_{p, j}\right)_{j \in \omega}$ by letting

$$
\begin{aligned}
m_{0,0} & =b \circ \$(\emptyset), & & \\
m_{p+1, j} & =b \circ \$\left(m_{p, 0}, \ldots, m_{p, j}\right) & & \text { for } p<3 n, \quad \text { and } \\
m_{0, j+1} & =b \circ \$\left(m_{3 n, 0}, \ldots, m_{3 n, j}\right) & & \text { for } j \in \omega .
\end{aligned}
$$

The sequence $\left(m_{p, j}\right)_{j \in \omega}$ will be interpreted as the moves of the second player in the $p$ th game.

It follows from our assumption on $b$ and $\$$ that

$$
m_{0,0}<m_{1,0}<\cdots<m_{3 n, 0}<m_{0,1}<m_{1,1}<\cdots<m_{3 n, 1}<m_{0,2}<\cdots .
$$

Let $M_{3 n}=\bigcup_{j \in \omega}\left[m_{3 n, j}, m_{0, j+1}\right)$ and $M_{p}=\bigcup_{j \in \omega}\left[m_{p, j}, m_{p+1, j}\right)$ for $0 \leq p<3 n$.

Since $M_{0} \cup \ldots \cup M_{3 n}=\omega$ for every $i \in\{1, \ldots, n\}$ there is a number $p(i) \epsilon$ $\{0, \ldots, 3 n\}$ such that $\left|M_{p(i)} \cap F\right|=\infty$ for each $F \in \mathscr{F}_{i}$. It follows by an elementary combinatorial argument that there is a number $p \in\{1, \ldots, 3 n-1\}$ such that $|p-p(i)|>1$ for every $i \in\{1, \ldots, n\}$.

We claim that the moves $\left(m_{p, j}\right)_{j \in \omega}$ of the second player beat the strategy $\$$ of I in the game $\mathrm{LH}_{[\omega]^{\omega}}$ on $U$. Let $n_{k+1}=\$\left(m_{p, 0}, \ldots, m_{p, k}\right)$ for $k \geq \emptyset$. To show that the strategy $\$$ is not winning, it suffices to find for each $x \in U$ an infinite subset $\mathscr{I} \subset \omega$ such that $\|x\|_{n_{k}} \leq m_{p, k}$ for all $k \in \mathscr{I}$.

Given arbitrary $x \in U$, find $i \in\{1, \ldots, n\}$ such that $x \in \circledast\left(b ; \mathscr{F}_{i}\right)$. Next, find an element $F_{i} \in \mathscr{F}_{i}$ such that $\|x\|_{j}<b(j)$ for all $j \in F_{i}$. It follows from the choice of the number $p$ that the set $\mathscr{I}=\left\{k \in \omega: F_{i} \cap\left[m_{p+1, k-1}, m_{p-1, k}\right) \neq \emptyset\right\}$ is infinite. 
We claim that $\|x\|_{n_{k}} \leq m_{p, k}$ for each $k \in \mathscr{I}$. Indeed, given any $k \in \mathscr{I}$, find a number $j \in F_{i} \cap\left[m_{p+1, k-1}, m_{p-1, k}\right)$. Note that

$$
n_{k}=\$\left(m_{p, 0}, \ldots, m_{p, k-1}\right) \leq b \circ \$\left(m_{p, 0}, \ldots, m_{p, k-1}\right)=m_{p+1, k-1} .
$$

Then

$$
\begin{aligned}
\|x\|_{n_{k}} & \leq\|x\|_{m_{p+1, k-1}} \leq\|x\|_{j}<b(j) \leq b\left(m_{p-1, k}\right) \\
& \leq b \circ \$\left(m_{p-1,0}, \ldots, m_{p-1, k}\right)=m_{p, k} .
\end{aligned}
$$

PROOF OF PROPOSITION 1. (1) To see that $\circledast\left([\omega]^{\omega}\right)$ is a dense $G_{\delta}$-subset of $\mathbb{R}^{\omega}$, notice that $\mathbb{R}^{\omega} \backslash \circledast\left([\omega]^{\omega}\right)=\bigcup_{n \in \omega} M_{n}$, where the sets $M_{n}$, defined by $M_{n}=\left\{x \in \mathbb{R}^{\omega}\right.$ : $|x(i)| \geq i$ for all $i \geq n\}$ for all $n \in \omega$, are closed nowhere dense subsets of $\mathbb{R}^{\omega}$.

(2) Theorem 2 implies that the set $\circledast\left([\omega]^{\omega}\right)$ is $o_{[\omega]^{\omega}}$-bounded and hence $o$-bounded.

(3) The set $\circledast\left([\omega]^{\omega}\right)$, being a dense $G_{\delta}$-subset of $\mathbb{R}^{\omega}$, is not $\sigma$-bounded, because $\sigma$-bounded subsets of $\mathbb{R}^{\omega}$ are meager. By Theorem 1 (2), I-bounded analytic subsets of $\mathbb{R}^{\omega}$ are $\sigma$-bounded. Consequently, $\circledast\left([\omega]^{\omega}\right)$, being analytic and not $\sigma$-bounded, is not I-bounded in $\mathbb{R}^{\omega}$.

(4) Assuming that $\circledast\left([\omega]^{\omega}\right)$ is $o_{\mathscr{F}}$-bounded for some filter $\mathscr{F}$, and applying Theorem 7 , we conclude that $\circledast\left([\omega]^{\omega}\right)$ is $I_{\mathscr{F}}$-bounded and thus $I$-bounded, which contradicts the previous item.

(5) If $B$ is a $\sigma$-bounded subset of $\mathbb{R}^{\omega}$, then the product $B \times \circledast\left([\omega]^{\omega}\right)$ is $o$-bounded in $\mathbb{R}^{\omega} \times \mathbb{R}^{\omega}$, by [11, Theorem 2.7] or Theorem 9 (2) applied to the near coherent semi-filters $[\omega]^{\omega}$ and $\mathfrak{F}_{r}$. Next, suppose that the product $B \times \circledast\left([\omega]^{\omega}\right)$ is $o$-bounded for some subset $B \subset \mathbb{R}^{\omega}$. Let $\Psi: \mathbb{R}^{\omega} \times \mathbb{R}^{\omega} \rightarrow \mathbb{R}^{\omega}$ be the isomorphism mapping a pair of sequences $\left(\left(x_{i}\right),\left(y_{i}\right)\right) \in \mathbb{R}^{\omega} \times \mathbb{R}^{\omega}$ onto the sequence $\left(z_{i}\right)$ such that $z_{2 i}=x_{i}$ and $z_{2 i+1}=y_{i}$ for $i \in \omega$. Then the image $\Psi\left(B \times \circledast\left([\omega]^{\omega}\right)\right)$ is $o$-bounded in $\mathbb{R}^{\omega}$. Applying Theorem 2, we conclude that the set $\Psi\left(B \times \circledast\left([\omega]^{\omega}\right)\right)$ is $o_{[\omega]^{\omega}}$-bounded and lies in $\circledast\left(f ;[\omega]^{\omega}\right)$ for some increasing function $f \in \omega^{\omega}$. Consider the increasing functions $g, h: \omega \rightarrow \omega$ defined by $g(i)=f(2 i+2)$ and $h(i)=g(g(i)+1)$ for $i \in \omega$.

The $\sigma$-boundedness of $B$ will follow as soon as we prove that $\uparrow b \leq^{*} h$ for any $b \in B$. Assuming that this is false, we would find a function $b \in B$ and an infinite subset $N \subset \omega$ such that $\|b\|_{n}>h(n)=g(g(n)+1)$ for every $n \in N$. Without loss of generality we can assume that $N \cap[n, g(n)]=\{n\}$ for any $n \in N$. Let $\left\{n_{k}\right\}_{k \in \omega}$ be the increasing enumeration of the set $N$. Consider the function $c \in \omega^{\omega}$ defined by $c(i)=\min (g(N) \cap[i-1, \infty))$ and observe that $c \in \circledast\left([\omega]^{\omega}\right)$. Let us show that $\max \left\{\|b\|_{i},\|c\|_{i}\right\} \geq g(i)$ for any $i \geq n_{0}$. Indeed, given arbitrary $i \geq n_{0}$, find a unique number $k$ such that $n_{k} \leq i<n_{k+1}$. The choice of the set $N$ implies that $g\left(n_{k}\right)<n_{k+1}$. If $i \leq g\left(n_{k}\right)+1$, then

$$
\max \left\{\|b\|_{i},\|c\|_{i}\right\} \geq\|b\|_{i} \geq\|b\|_{n_{k}}>g\left(g\left(n_{k}\right)+1\right) \geq g(i),
$$


while if $i>g\left(n_{k}\right)+1$, then

$$
\begin{aligned}
\max \left\{\|b\|_{i},\|c\|_{i}\right\} & \geq\|c\|_{i}=\min g(N) \cap[i-1, \infty) \\
& \geq \min g(N) \cap\left(g\left(n_{k}\right), \infty\right)=g\left(n_{k+1}\right) \geq g(i) .
\end{aligned}
$$

Consider the function $a=\Psi(b, c)$, and note that

$$
a \in \Psi\left(B \times \circledast\left([\omega]^{\omega}\right) \subset \circledast\left(f ;[\omega]^{\omega}\right) .\right.
$$

On the other hand, given any $k>2 n_{0}$, find the smallest $i \in \omega$ with $2 i>k$, and observe that $i \geq n_{0}$ and hence $\|a\|_{k} \geq \max \left\{\|b\|_{i},\|c\|_{i}\right\} \geq g(i)=f(2 i+2) \geq f(k)$. Now the inequality $f \leq^{*} \uparrow a$ contradicts the fact that $a \in \circledast\left(f,[\omega]^{\omega}\right)$. This contradiction completes the proof of the $\sigma$-boundedness of $B$.

ProOF OF THEOREM 9. We shall prove the implications (1) implies (2) implies (3) implies (4) implies (5) implies (6) implies (1), (1) implies (7) implies (8) implies (1), and (1) implies (9) implies (4). In fact, the implications (7) implies (8) and (2) implies (3) implies (4) are trivial. To see (4) implies (5) note that $\circledast\left(\mathscr{F}_{1}\right)+\cdots+\circledast\left(\mathscr{F}_{n}\right)$ is the image of $\circledast\left(\mathscr{F}_{1}\right) \times \cdots \times \circledast\left(\mathscr{F}_{n}\right)$ under the continuous group homomorphism $h:\left(\mathbb{R}^{\omega}\right)^{n} \rightarrow \mathbb{R}^{\omega}, h:\left(x_{1}, \ldots, x_{n}\right) \mapsto x_{1}+\cdots+x_{n}$, and use a result of [10] asserting that $o$-bounded groups are preserved by homomorphic images.

(1) implies (2): Assume that the (semi-)filters $\mathscr{F}_{1}, \ldots, \mathscr{F}_{n}$ are near coherent, and let $b \in \omega^{\omega}$ be an increasing function. Using the near coherence of the semi-filters $\mathscr{F}_{1}, \ldots, \mathscr{F}_{n}$, find an increasing sequence $\left(m_{k}\right)_{k \in \omega}$ such that $m_{0}=0$ and such that for any $F_{i} \in \mathscr{F}_{i}$, for $1 \leq i \leq n$, the set

$$
\mathscr{I}\left(F_{1}, \ldots, F_{n}\right)=\left\{k \in \omega:\left[m_{k}, m_{k+2}\right) \cap F_{i} \neq \emptyset \text { for all } i \leq n\right\}
$$

is non-empty. Consider the (semi-)filter

$$
\mathscr{F}=\left\{F \subset \omega: \bigcup_{k \in \mathscr{I}\left(F_{1}, \ldots, F_{n}\right)}\left[n m_{k}, n m_{k+1}\right) \subset F \text { for some } F_{1} \in \mathscr{F}_{1}, \ldots, F_{n} \in \mathscr{F}_{n}\right\} .
$$

Next, define the function $f: \omega \rightarrow \omega$ by $f: j \mapsto b\left(m_{k+2}\right)$, where $k$ is chosen to satisfy the condition $j \in\left[n m_{k}, n m_{k+1}\right)$.

Consider the linear isomorphism $\Psi: \mathbb{R}^{\omega} \rightarrow \mathbb{R}^{n}$ assigning to an $n$-tuple of functions $\left(x_{1}, \ldots, x_{n}\right) \in \mathbb{R}^{\omega}$ the function $y=\Psi\left(x_{1}, \ldots, x_{n}\right)$ defined by $y(n q+i)=x_{i+1}(q)$ for all $q \in \omega$ and $0 \leq i<n$. We claim that $\Psi\left(\circledast\left(b ; \mathscr{F}_{1}\right) \times \cdots \times \circledast\left(b ; \mathscr{F}_{n}\right)\right) \subset \circledast(f ; \mathscr{F})$. Take any functions $x_{i} \in \circledast\left(b ; \mathscr{F}_{i}\right)$ for $1 \leq i \leq n$, and let $y=\Psi\left(x_{1}, \ldots, x_{n}\right)$. For every $i$, find $F_{i} \in \mathscr{F}_{i}$ such that $\left\|x_{i}\right\|_{j}<\|b\|_{j}$ for all $j \in F_{i}$, and let

$$
F=\bigcup_{k \in \mathscr{I}\left(F_{1}, \ldots, F_{n}\right)}\left[n m_{k}, n m_{k+1}\right) \in \mathscr{F}
$$


We claim that $\|y\|_{j}<\|f\|_{j}$ for all $j \in F$. We have to verify that $|y(p)|<\|f\|_{j}$ for every $p \leq j$. Write $p=n q+i$, where $q \in \omega$ and $0 \leq i<n$. Next, find $k \in \omega$ such that $j \in\left[n m_{k}, n m_{k+1}\right)$. Since $p \leq j$, we have $q \leq m_{k}$. Because $k \in \mathscr{I}\left(F_{1}, \ldots, F_{n}\right)$, there is a point $q^{\prime} \in F_{i+1} \cap\left[m_{k}, m_{k+2}\right)$. Then $q \leq m_{k} \leq q^{\prime}<m_{k+2}$ and

$$
|y(p)|=\left|x_{i+1}(q)\right| \leq\left\|x_{i+1}\right\|_{q^{\prime}}<\|b\|_{q^{\prime}}=b\left(q^{\prime}\right) \leq b\left(m_{k+2}\right)=f(j) \leq\|f\|_{j} .
$$

Thus $y \in \circledast(f ; \mathscr{F})$ and $\Psi\left(\prod_{i=1}^{n} \circledast\left(b ; \mathscr{F}_{i}\right)\right) \subset \circledast(f ; \mathscr{F})$. By Theorem 2 , the set $\circledast(f ; \mathscr{F})$ is $o_{\mathscr{F}}$-bounded in $\mathbb{R}^{\omega}$. Since $\Psi$ is a group isomorphism, the product $\circledast\left(b ; \mathscr{F}_{1}\right) \times \cdots \times \circledast\left(b ; \mathscr{F}_{n}\right)$ and its subset $\circledast\left(B_{1} ; \mathscr{F}_{1}\right) \times \cdots \times \circledast\left(B_{n} ; \mathscr{F}_{n}\right)$ are therefore $o_{\mathscr{F}}$-bounded in $\mathbb{R}^{\omega}$.

(6) implies (1): Assume that $\circledast\left(\mathscr{F}_{1}\right)+\cdots+\circledast\left(\mathscr{F}_{n}\right) \neq \mathbb{R}^{\omega}$. First we show that the set $\circledast\left(\mathscr{F}_{1}\right)+\cdots+\circledast\left(\mathscr{F}_{n}\right)$ is absolutely symmetric in $\mathbb{R}^{\omega}$. Fix any $x \in \circledast\left(\mathscr{F}_{1}\right)+\cdots+\circledast\left(\mathscr{F}_{n}\right)$ and any $y \in \mathbb{R}^{\omega}$ with $\uparrow y \leq \uparrow x$. Write $x=x_{1}+\cdots+x_{n}$, where $x_{i} \in \circledast\left(\mathscr{F}_{i}\right)$, $i \leq n$. Then $|y| \leq \uparrow y \leq \uparrow x \leq \uparrow x_{1}+\cdots+\uparrow x_{n}$, and we can find functions $y_{1}, \ldots, y_{n} \in \mathbb{R}^{\omega}$ such that $y=y_{1}+\cdots+y_{n}$ and $\left|y_{i}\right| \leq \uparrow x_{i}$ for all $i \leq n$. By the absolute symmetry of the sets $\circledast\left(\mathscr{F}_{i}\right)$, we have $y_{i} \in \circledast\left(\mathscr{F}_{i}\right)$ for all $i$, and thus $y=y_{1}+\cdots+y_{n} \in \circledast\left(\mathscr{F}_{1}\right)+\cdots+\circledast\left(\mathscr{F}_{n}\right)$.

Fix any $f \notin \circledast\left(\mathscr{F}_{1}\right)+\cdots+\circledast\left(\mathscr{F}_{n}\right)$. Because the set $\circledast\left(\mathscr{F}_{1}\right)+\cdots+\circledast\left(\mathscr{F}_{n}\right)$ is absolutely symmetric, we can assume that $f=\uparrow f$ and that $f$ is thus a positive non-decreasing function. Let $m_{0}=0$ and $m_{k+1}=f\left(m_{k}\right)+1$ for $k \in \omega$. We claim that for any sets $F_{1} \in \mathscr{F}_{1}, \ldots, F_{n} \in \mathscr{F}_{n}$, there is a $k \in \omega$ such that $\left[m_{k}, m_{k+2}\right) \cap F_{i} \neq \emptyset$ for all $i \leq n$. Without loss of generality, $0 \notin F_{i}$ for all $i \leq n$.

For every $1 \leq i \leq n$, consider the function $x_{i}=\mathrm{id}_{F_{i}}-1 \in \circledast\left(\mathscr{F}_{i}\right)$, by which notation we mean the function $x_{i}: j \mapsto \min \left(F_{i} \cap[j, \infty)\right)-1$. Then the function $y=x_{1}+\cdots+x_{n}$ belongs to $\circledast\left(\mathscr{F}_{1}\right)+\cdots+\circledast\left(\mathscr{F}_{n}\right)$. As $f \notin \circledast\left(\mathscr{F}_{1}\right)+\cdots+\circledast\left(\mathscr{F}_{n}\right)$, we conclude that $\uparrow y(j)<\uparrow f(j)$ for some $j \in \omega$. Find $k \in \omega$ such that $j \in\left[m_{k}, m_{k+1}\right)$. Then for any $1 \leq i \leq n$, we have

$$
\begin{aligned}
\min \left(F_{i} \cap[j, \infty)\right)-1 & =x_{i}(j) \leq \uparrow y(j)<\uparrow f(j) \\
& =f(j) \leq f\left(m_{k+1}\right)=m_{k+2}-1 .
\end{aligned}
$$

Hence $\min \left(F_{i} \cap\left[m_{k}, \infty\right)\right) \leq \min \left(F_{i} \cap[j, \infty)\right)<m_{k+2}$ and $F_{i} \cap\left[m_{k}, m_{k+2}\right) \neq \emptyset$ for all $1 \leq i \leq n$. This means that the semi-filters $\mathscr{F}_{1}, \ldots, \mathscr{F}_{n}$ are near coherent.

(1) implies (7): Assume that $\mathscr{F}_{1}, \ldots, \mathscr{F}_{n}$ are near coherent filters, and fix an increasing number sequence $\left(m_{k}\right)$ such that for any $F_{1} \in \mathscr{F}_{1}, \ldots ; F_{n} \in \mathscr{F}_{n}$ there is a $k \in \omega$ such that $\left[m_{k}, m_{k+2}\right) \cap F_{i} \neq \emptyset$ for every $i \leq n$. For every permutation $\sigma$ of the set $\{1, \ldots, n\}$ and elements $F_{i} \in \mathscr{F}_{i}$, for $i \leq n$, let

$$
\begin{aligned}
& K_{\sigma}\left(F_{\sigma(1)}, \ldots, F_{\sigma(n)}\right) \\
& \quad=\left\{m_{k}: \exists p_{1} \geq \cdots \geq p_{n} \text { with } p_{i} \in\left[m_{k}, m_{k+2}\right) \cap F_{\sigma(i)} \text { for } 1 \leq i \leq n\right\},
\end{aligned}
$$


and also let $\mathscr{F}_{\sigma}=\left\{K_{\sigma}\left(F_{\sigma(1)}, \ldots, F_{\sigma(n)}\right): F_{i} \in \mathscr{F}_{i}\right.$ for $\left.1 \leq i \leq n\right\}$. It is easy to see that for some permutation $\sigma$ of $\{1, \ldots, n\}$ the collection $\mathscr{F}_{\sigma}$ is centered, and hence can be completed to a filter $\mathscr{F}$. (A collection $\mathscr{A}$ is centered if $\cap \mathscr{C} \neq \emptyset$ for any finite subcollection $\mathscr{C} \subset \mathscr{A})$.

We shall show that the set $\circledast\left(b ; \mathscr{F}_{\sigma(1)}, \ldots, \mathscr{F}_{\sigma(n)}\right)$ is $o_{\mathscr{F}}$-bounded for this permutation $\sigma$ and for any function $b \in \mathbb{R}^{\omega}$. To do this, fix any increasing function $f: \omega \rightarrow \omega$ such that $f\left(m_{k}\right) \geq\|b\|_{m_{k+2}}$. We claim that $\circledast\left(b ; \mathscr{F}_{\sigma(1)}, \ldots, \mathscr{F}_{\sigma(n)}\right) \subset \circledast(f ; \mathscr{F})$.

Fix any function $x \in \circledast\left(b ; \mathscr{F}_{\sigma(1)}, \ldots, \mathscr{F}_{\sigma(n)}\right)$. Let $x_{n}=x$, and find by induction, for every $i \in\{n, n-1, \ldots, 2\}$, a function $x_{i-1} \in \circledast\left(b ; \mathscr{F}_{\sigma(1)}, \ldots, \mathscr{F}_{\sigma(i-1)}\right)$ and an element $F_{\sigma(i)} \in \mathscr{F}_{\sigma(i)}$ such that $\left\|x_{i}\right\|_{j}<\left\|x_{i-1}\right\|_{j}$ for every $j \in F_{\sigma(i)}$. Finally, find a set $F_{\sigma(1)} \in \mathscr{F}_{\sigma(1)}$ such that $\left\|x_{1}\right\|_{j}<\|b\|_{j}$ for every $j \in F_{\sigma(1)}$.

We claim that $\|x\|_{m_{k}}<f\left(m_{k}\right)$ for every $m_{k} \in K_{\sigma}\left(F_{\sigma(1)}, \ldots, F_{\sigma(n)}\right) \in \mathscr{F}$. Indeed, given such an $m_{k}$, we can find numbers $p_{1} \geq \cdots \geq p_{n}$ such that $p_{i} \in\left[m_{k}, m_{k+2}\right) \cap F_{\sigma(i)}$ for every $1 \leq i \leq n$. Then

$$
\begin{aligned}
\|x\|_{m_{k}} & \leq\left\|x_{n}\right\|_{p_{n}}<\left\|x_{n-1}\right\|_{p_{n}} \leq\left\|x_{n-1}\right\|_{p_{n-1}} \\
& <\left\|x_{n-2}\right\|_{p_{n-2}} \leq \cdots \leq\left\|x_{1}\right\|_{p_{1}}<\|b\|_{p_{1}} \leq\|b\|_{m_{k+2}} \leq f\left(m_{k}\right),
\end{aligned}
$$

and thus $x \in \circledast(f, \mathscr{F})$. By Theorem 2 , the set $\circledast\left(b ; \mathscr{F}_{\sigma(1)}, \ldots, \mathscr{F}_{\sigma(n)}\right)$, being a subset of $\circledast(f ; \mathscr{F})$, is $o_{\mathscr{F}}$-bounded.

(8) implies (1): Assume that $\circledast\left(\mathscr{F}_{\sigma(1)}, \ldots, \mathscr{F}_{\sigma(n)}\right) \neq \mathbb{R}^{\omega}$ for some permutation $\sigma$ of $\{1, \ldots, n\}$. We have to show that the semi-filters $\mathscr{F}_{1}, \ldots \mathscr{F}_{n}$ are near coherent. Without loss of generality, assume that $\sigma(i)=i$ for every $i$.

Fix any function $f \notin \circledast\left(\mathscr{F}_{1}, \ldots, \mathscr{F}_{n}\right)$. Since the set $\circledast\left(\mathscr{F}_{1}, \ldots, \mathscr{F}_{n}\right)$ is absolutely symmetric, we can assume that $f$ is increasing and takes positive integer values. Let $m_{0}=0$ and $m_{k+1}=f\left(m_{k}\right)+2$ for $k \geq 0$. The near coherence of the filters $\mathscr{F}_{1}, \ldots, \mathscr{F}_{n}$ will follow as soon as we show that for any $F_{1} \in \mathscr{F}_{1}, \ldots, F_{n} \in \mathscr{F}_{n}$ there is a number $k \in \omega$ such that $\left[m_{k}, m_{k+2}\right) \cap F_{i} \neq \emptyset$ for every $i \leq n$.

Consider the sequence of increasing functions $g_{0}, \ldots, g_{n} \in \mathbb{R}^{\omega}$, where $g_{0}(j)=j$ and $g_{i}(j)=-1 / n+\min F_{n} \cap\left[g_{i-1}(j), \infty\right)$ for $j \in \omega$. It is easy to see that $g_{i} \in \circledast\left(\mathscr{F}_{1}, \ldots, \mathscr{F}_{i}\right)$ for every $i \leq n$. It follows from $f \notin \circledast\left(\mathscr{F}_{1}, \ldots, \mathscr{F}_{n}\right) \ni g_{n}$ that $g_{n}(j) \leq f(j)$ for some $j \in \omega$. For this number $j$, find a unique $k$ such that $j \in\left[m_{k}, m_{k+1}\right)$.

It follows from the definition of the number $g_{n}(j) \leq f(j)$ that there is a number $j_{n} \in F_{n}$ such that $g_{n-1}(j) \leq j_{n} \leq f(j)+1 / n$. Similarly, for the function $g_{n-1}$ there is a number $j_{n-1} \in F_{n-1}$ such that $g_{n-2}(j) \leq j_{n-1} \leq j_{n}+1 / n \leq f(j)+2 / n$. Proceeding in this way, for every $i \in\{n, n-1, \ldots, 1\}$ we find a number $j_{i} \in F_{i}$ such that $g_{i-1}(j) \leq j_{i} \leq f(j)+(n-i+1) / n$. Then

$$
m_{k} \leq j=g_{0}(j) \leq j_{1}, \ldots, j_{n} \leq f(j)+1 \leq f\left(m_{k+1}\right)+1<m_{k+2},
$$

and thus each set $F_{i}$, for $1 \leq i \leq n$, meets the half-interval $\left[m_{k}, m_{k+2}\right)$. 
(1) implies (9): If the filters $\mathscr{F}_{1}, \ldots, \mathscr{F}_{n}$ are near coherent, then the product $\circledast\left(\mathscr{F}_{1}\right) \times \cdots \times \circledast\left(\mathscr{F}_{n}\right)$ is $o_{\mathscr{F}}$-bounded in $\left(\mathbb{R}^{\omega}\right)^{n}$ for some filter $\mathscr{F}$, by the implication (1) implies (2). Hence the image $\circledast\left(\mathscr{F}_{1}\right)+\cdots+\circledast\left(\mathscr{F}_{n}\right) \supset \circledast\left(\mathscr{F}_{1}\right) \cup \cdots \cup \circledast\left(\mathscr{F}_{n}\right)$ of this product under the continuous homomorphism $h:\left(\mathbb{R}^{\omega}\right)^{n} \rightarrow \mathbb{R}^{\omega}$ defined by $h:\left(x_{1}, \ldots, x_{n}\right) \mapsto x_{1}+\cdots+x_{n}$ is also $o_{\mathscr{F}}$-bounded in $\mathbb{R}^{\omega}$.

(9) implies (4): Assume that the union $\circledast\left(\mathscr{F}_{1}\right) \cup \cdots \cup \circledast\left(\mathscr{F}_{n}\right)$ is $o_{\mathscr{F}}$-bounded from some filter $\mathscr{F}$. Then the sets $\circledast\left(\mathscr{F}_{1}\right), \ldots, \circledast\left(\mathscr{F}_{n}\right)$ are $o_{\mathscr{F}}$-bounded in $\mathbb{R}^{\omega}$, and hence their product $\circledast\left(\mathscr{F}_{1}\right) \times \cdots \times \circledast\left(\mathscr{F}_{n}\right)$ is $o$-bounded, according to the implication (1) implies (2) of Theorem 9 applied to $n$ copies of the filter $\mathscr{F}$.

PROOF OF PROPOSITION 2. Given an $o$-bounded mixable subset $X \subset \mathbb{R}^{\omega}$, find a nondecreasing function $f:[0, \infty) \rightarrow[0, \infty)$ such that for any $x, y \in X$ there is a $z \in X$ such that $f \circ \uparrow z \geq^{*} \max \{\uparrow x, \uparrow y\}$. Replacing $f(t)$ by $\max \{f(t), t\}$ if necessary, we can assume that $f(t) \geq t$ for all $t \in[0, \infty)$. Let $f^{1}=f$, and by recursion let $f^{n+1}=f \circ f^{n}$ for $n \geq 1$. Take any increasing function $g:[0, \infty) \rightarrow[0, \infty)$ such that $g \geq^{*} f^{n}$ for all $n \geq 1$. Then by induction it can be shown that for any $x_{1}, \ldots, x_{n} \in X$ there is a $z \in X$ such that $g \circ \uparrow z \geq^{*} \max \left\{\uparrow x_{1}, \ldots, \uparrow x_{n}\right\}$.

Using the $o$-boundedness of the set $X$ and Theorem 2, find a non-decreasing function $b \in \mathbb{R}^{\omega}$ such that $X \subset \circledast\left(b ;[\omega]^{\omega}\right)$. For each $x \in X$, let $F_{x}=\{n \in \omega$ : $\uparrow x(n)<g \circ b(n)\}$, and consider the collection $\mathscr{C}=\left\{F_{x}: x \in X\right\}$. We claim that this collection is centered. Indeed, assuming the converse to hold, we would find points $x_{1}, \ldots, x_{n} \in X$ such that $F_{x_{1}} \cap \cdots \cap F_{x_{n}}=\emptyset$. The last equality implies that $\max \left\{\uparrow x_{1}, \ldots, \uparrow x_{n}\right\} \geq g \circ b$. It follows from the choice of the function $g$ that there is an element $z \in X$ such that $g \circ \uparrow z \geq^{*} \max \left\{\uparrow x_{1}, \ldots, \uparrow x_{n}\right\} \geq g \circ b$. Taking into account that the map $g$ is increasing, we get $\uparrow z \geq^{*} b$, which contradicts $z \in \circledast\left(b ;[\omega]^{\omega}\right)$.

Therefore the collection $\mathscr{C}$ is centered, and can be completed to a filter $\mathscr{F} \supset \mathscr{C}$. Then $X \subset \circledast(g \circ b ; \mathscr{C}) \subset \circledast(g \circ b ; \mathscr{F})$, and by Theorem $2, X$ is $o_{\mathscr{F}}$-bounded.

\section{Acknowledgements}

The initial ideas for this paper were developed during a visit of the second author to the Departamento de Matemáticas of the Universidad Autónoma Metropolitana, Mexico City, during the first quarter of 2001, and visits of the first and second authors to the Departamento de Matemáticas of the Universidad Jaume I in Castellón, Spain, during the second quarter of 2001. These authors thank the respective departments for their hospitality. The second author also thanks Universidad Jaume I for its financial support under the research grant Programa de Movilidad de Personal Investigador Estancias Investigadores Extranjeros (Acción 2.2).

In addition, the authors would like to express their sincere thanks to Lubomyr Zdomsky and Henryk Michalewski for valuable and stimulating discussions, which 
resulted in essential improvements to the manuscript.

\section{References}

[1] T. Banakh, 'On index of total boundedness of (strictly) o-bounded groups', Topology Appl. 120 (2002), 427-439.

[2] T. Banakh and L. Zdomsky, 'Selection principles and games on multicovered spaces and their application in topological algebra', in preparation.

[3] T. Bartoszynski and H. Judah, 'Measure and category-filters on $\omega$ ', in: Set theory of the continuum, Math. Sci. Res. Inst. Publ. 26 (Springer, Berlin, 1992) pp. 175-202.

[4] T. Bartoszyński and H. Judah, Set theory: On the structure of the real line (A. K. Peters Ltd., Wellesley, Mass., 1995).

[5] A. Blass, 'Near coherence of filters I', Notre Dame J. Formal Logic 27 (1986), 579-591.

[6] - 'Near coherence of filters II', Trans. Amer. Math. Soc. 300 (1987), 557-581.

[7] A. Blass and S. Shelah, 'There may be simple $P_{\aleph_{1}}$ - and $P_{\aleph_{2}}$-points and the Rudin-Keisler ordering may be downward directed', Ann. Pure Appl. Logic 33 (1987), 213-243.

[8] _ - 'Near coherence of filters III: A simplified consistency proof', Notre Dame J. Formal Logic 30 (1989), 530-538.

[9] J. Chaber and R. Pol, 'A remark on Fremlin-Miller theorem concerning the Menger property and Michael concentrated sets', preprint.

[10] C. Hernández, 'Topological groups close to being $\sigma$-compact', Topology Appl. 102 (2000), $101-$ 111.

[11] C. Hernández, D. Robbie and M. Tkachenko, 'Some properties of $o$-bounded and strictly $o$-bounded groups', Appl. General Topology 1 (2000), 29-43.

[12] K. Hrbacek and T. Jech, Introduction to set theory (Marcel Dekker, New York, 1999).

[13] H. Judah and S. Shelah, 'Martin's axiom, measurability and equiconsistency results', New York J. Symbolic Logic 54 (1989), 78-94.

[14] A. Kechris, Classical descriptive set theory (Springer, Berlin, 1995).

[15] A. Krawczyk and H. Michalewski, 'An example of a topological group', Topology Appl. 127 (2003), 325-330.

[16] _..., 'Linear metric spaces close to being sigma-compact', preprint, (Inst. of Math., Warsaw Univ., 2001).

[17] C. Laflamme, 'Filter games and combinatorial properties of strategies', Contemp. Math. 192 (1996), 51-67.

[18] C. Laflamme and C. C. Leary, 'Filter games on $\omega$ and the dual ideal', Fund. Math. 173 (2002), $159-173$.

[19] D. A. Martin and J. R. Steel, 'A proof of projective determinacy', J. Amer. Math. Soc. 2 (1989), $71-125$.

[20] H. Michalewski, Przestrzenie funkcyjne z topologia zbieżnościpunktowej (Ph.D. Thesis, University of Warsaw, 2002), (in Polish).

[21] M. Talagrand, 'Compacts de fonctions mesurables et filtres non mesurables', Studia Math. 67 (1980), 13-43.

[22] M. Tkachenko, 'Introduction to topological groups', Topology Appl. 86 (1998), 179-231.

[23] _ _ 'Topological groups: between compactness and $\aleph_{0}$-boundedness', in: Recent progress in general topology II (eds. M. Hušek and J. van Mill) (North-Holland, 2002) pp. 515-543.

[24] S. Todorcevic, Topics in topology, Lecture Notes in Mathematics 1652 (Springer, Berlin, 1997). 
[25] B. Tsaban, ' $o$-Bounded groups and other topological groups with strong combinatorial properties', Proc. Amer. Math. Soc. 134 (2006), 881-891.

[26] J. van Mill, 'Introduction to $\beta \omega$ ', in: Handbook of set-theoretic topology (eds. K. Kunen and J. Vaughan) (North-Holland, Amstrerdam, 1984) Chapter 11, pp. 503-568.

Instytut Matematyki

Akademia Świętokrzyska

Świȩtokrzyska 15

Kielce

Poland

and

Department of Mathematics

Ivan Franko Lviv National University

Universytetska 1

Lviv 79000

Ukraina

e-mail: tbanakh@franko.lviv.ua

Departament de Matemàtiques

Universitat Jaume I

Campus de Penyeta Roja

s/n 12071

Castellón

Spain

e-mail: sanchis@mat.uji.es
School of Mathematics and Applied Statistics University of Wollongong Wollongong NSW 2522

Australia e-mail: peter@uow.edu.au 